Cita bibliográfica: Gutiérrez-Fernandez, L. F., Martínez-Daza, S., Gómez Acosta, C., Gil Perez, V. y Cabezas Pinzón, L. (2021). Cálculo de la capacidad de carga y capacidad de acogida turística multicriterio para la reserva biológica El Encenillo, Guasca, Cundinamarca, Colombia. Investigaciones Turísticas (21), pp. 224-255. https://doi. org/10.14198/INTURI2021.21.11

\title{
Cálculo de la capacidad de carga y capacidad de acogida turística multicriterio para la reserva biológica El Encenillo, Guasca, Cundinamarca, Colombia
}

\section{Calculation of the carrying capacity and multi-criteria tourist reception capacity for the El Encenillo biological reserve, Guasca-Cundinamarca, Colombia}

Luis Fernando Gutiérrez-Fernandez iD, Universidad del Bosque, Colombia gutierrezluisf@unbosque.edu.co

Sofía Martínez-Daza (iD, Universidad del Bosque, Colombia smartinezdaz@unbosque.edu.co

Camilo Gómez Acosta iD, Universidad del Bosque, Colombia cgomezac@unbosque.edu.co

Verónica Gil Perez (iD, Universidad del Bosque, Colombia vgil@unbosque.edu.co

Laura Cabezas Pinzón (iD, Universidad del Bosque, Colombia Icabezasp@uelbosque.edu.co

\section{RESUMEN}

La presente investigación tiene como objetivo principal realizar una comparación entre dos metodologías para calcular el número máximo de visitantes que pueden ingresar diariamente a un área natural en donde se realiza turismo, para establecer las fortalezas y debilidades de cada una. Como caso de estudio se seleccionó la reserva Biológica El Encenillo ubicada en el municipio de Guasca, departamento de Cundinamarca - Colombia. Las metodologías utilizadas fueron la Capacidad De Carga Turística (CCT), ampliamente utilizada, pero que en la actualidad ha ido cayendo en desuso y se le tacha de subjetiva y el Cálculo de Acogida Turística Multicriterio - CATUM, que sigue un método basado en la ponderación de los factores de corrección mediante el Proceso Analítico Jerárquico (Analytic Hierarchy Process - AHP), aplicado en 3 niveles de consulta: Sui validatio, Scientatis validatio y Societatis validatio, como mecanismo para reducir la subjetividad. 
Palabras clave: Capacidad de carga; capacidad de acogida, análisis multicriterio, turismo sostenible.

\section{ABSTRACT}

The main objective of this research is to carry out a comparison between two methodologies to calculate the maximum daily number of visitors that can enter a natural area where tourism is carried out, to establish the strengths and weaknesses of each of them. The biological reserve El Encenillo was selected as a case study. It is located in the municipality of Guasca, department of Cundinamarca - Colombia. The methodologies used were the Tourist Carrying Capacity (CCT), which was formerly widely used, but has now fallen into disuse and is considered to be subjective, and the Multi-criteria Tourism Hosting Calculation, which follows a method based on the weighting of correction factors through the Analytic Hierarchy Process (AHP), applied at 3 levels of consultation: Sui validatio, Scientatis validatio and Societatis validatio, as a mechanism to reduce subjectivity.

Keywords: Carrying capacity, reception capacity, multicriteria analysis, sustainable tourism.

\section{INTRODUCCIÓN}

En los últimos años el aumento del turismo en Colombia ha sido sostenido y, de acuerdo con su Ministerio de Comercio Industria y Turismo, el crecimiento del país es cuatro veces mayor que el promedio mundial, lo que demuestra el buen momento del sector; sin embargo, se requiere de su planificación, especialmente cuando el mayor potencial lo representa el turismo de naturaleza.

El turismo, como lo expone la política colombiana para el desarrollo del ecoturismo, “(...) en cierto momento, fue considerado como el factor depredador número uno y, por tanto, el enemigo declarado de los recursos naturales" (República de Colombia et al, 2003. p. 12). Sin embargo, hoy se enfoca en una gestión integral de manera que se garanticen las necesidades económicas, sociales y estéticas, y se respete la integridad cultural, los procesos ecológicos esenciales, la diversidad biológica y los sistemas de soporte de la vida (Tapia, 2014). "Las preguntas clave siempre han sido ¿Cuántos son demasiados?, ¿Cómo se puede determinar esto?" (Wall, 2020. p. 213); son varias las metodologías que se han utilizado para analizar los territorios que cuentan con un alto capital natural y que son sometidos a un uso turístico en ocasiones intensivo, lo que origina transformaciones, conflictos y externalidades que ponen en duda su continuidad a largo plazo; no obstante, la Capacidad de Carga Turística (CCT) es una de las que mayor espectro de uso presenta.

El concepto de capacidad de carga es un concepto de la biología descrito como "la capacidad de un ecosistema para sustentar organismos sanos y, al mismo tiempo, mantener su productividad, adaptabilidad y capacidad de renovación" (Canadian Arctic Resources Committee, citado en Maggi \& Fredella, 2010. p. 2). Anteriormente "la OMT (1981) la había definido como el número máximo de personas que pueden visitar un lugar al mismo tiempo, sin causar la destrucción del medio físico, económico, socio-cultural y una disminución 
inaceptable en la calidad de la satisfacción de los visitantes" (PAP/RAC, 1997. p.5); lo cual concuerda con autores que aseveran que los estudios de capacidad de carga constituyen uno de los primeros intentos de operativizar el concepto de sostenibilidad en relación con la gestión de la actividad turística y garantizar la calidad de los espacios turísticos y su función recreativa (Bertoni et al, 2016).

No obstante lo anterior, en la actualidad las dinámicas turísticas desde la formulación de la metodología por parte de O'Reilly (1986) y popularizada por Cifuentes (1990; 1992a,b; 1999), han presentado cambios considerables; por lo tanto, se requiere adoptar nuevas herramientas y modelos que prevengan los excesos y el mal uso de los recursos naturales de los territorios turísticos, de forma que se genere una competitividad de estos espacios, entendida ésta como la capacidad de mejorar e innovar constantemente para generar ventajas diferenciales frente a los competidores, lo cual implica realizar un análisis estructural de los mercados para elegir las alternativas más viables (Porter, 2010), de manera que se pueda lograr un equilibrio ente los intereses generalmente opuestos que presentan las reservas naturales de uso y conservación.

Por lo anterior, la presente investigación propone realizar la comparación entre los resultados del cálculo de la capacidad de carga medida de forma tradicional (CCT) y los obtenidos a través de la metodología de Capacidad de Acogida Multicriterio (CATUM) propuesta realizada por Gutiérrez-Fernández \& Torres (2019), con el fin de analizar las ventajas y debilidades de cada uno de los modelos y que tanto se acercan los valores resultantes al manejo que se le está dando por parte de los administradores del área. Para el estudio se seleccionó "El Encenillo" que es una Reserva Biológica ubicada en el municipio de Guasca, departamento de Cundinamarca -Colombia.

La selección de la Reserva Biológica Encenillo como caso de estudio se debió a que es un espacio destinado a la protección y preservación de los bosques principalmente de encenillo (Weinmannia tomentosa), con unos objetivos claros de conservación del ecosistema, pero que al ser un lugar privado requiere de recursos económicos para su funcionamiento y por ello ve en el turismo una opción económicamente viable. Sin embargo, debido a la alta cantidad de visitantes que recibe al año, es posible que se vean afectados o ralentizados los procesos de sucesión ecológica y que sea más lento el alcance del clímax ecológico en aquellas zonas donde se presenta un mayor flujo de turistas, que finalmente es el objetivo principal que persigue la reserva. Por lo tanto, es necesario que se planifiquen las actividades asociadas a la actividad turística, de forma que se alcance un desarrollo sostenible, logrando así fortalecer las tres dimensiones del mismo (ecológica, social y económica).

\section{EXPLICACIÓN DE LA CAPACIDAD DE CARGA TURÍSTICA (CCT) Y LA CAPACIDAD DE ACOGIDA MULTICRITERIO (CATUM)}

\subsection{Capacidad de Carga Turística (CCT)}

La capacidad de carga es un instrumento de medición que presenta un rango de valores los cuales deben encontarse asociados a los objetivos de manejo específicos para un área y 
donde los efectos ambientales no solo dependen de la actividad que se realice, sino también de la fragilidad o de la capacidad de absorción del medio (Graefe et al, 1990).

La Capacidad de Carga Turística (CCT) como se mencionó en la parte introductoria, fue propuesta por primera vez por O'Reilly (1986) y popularizada posteriormente por Miguel Cifuentes (1990; 1992a,b; 1999) y ha sido ampliamente utilizada desde ese momento, encontrándose recientemente los trabajos de: Trumbic (2017); Montalvo \& Castillo (2018); Sati (2018); Ortega et al, (2020); para el caso colombiano se encuentran los trabajos de: Botero et al, (2008); Álvarez, M. P. (2010); Morales-Betancourt, (2014); Gutiérrez-Fernández \& Sierra, (2015).

De acuerdo a la metodología empleada por Cifuentes, el número máximo de visitas que un área puede recibir, debe tener en cuenta las condiciones físicas, biológicas y de gestión de la zona y consiste en tres fases: Capacidad de Carga Física (CCF), Capacidad de Carga Real (CCR), y Capacidad de Carga Efectiva (CCE); para calcular esta última se requiere haber calculado previamente la Capacidad de Carga de Manejo (CCM). A continuación, se describen las capacidades de carga:

Capacidad de Carga Física (CCF): “Es el límite máximo de visitas que se pueden hacer al sitio durante un día. Está dada por la relación entre factores de visita (horario y tiempo de visita), el espacio disponible y la necesidad de espacio por visitante" (Cifuentes et al., 1999, p.16).

Capacidad de Carga Real (CCR): "Es el límite máximo de visitas, determinado a partir de la CCF de un sitio, luego de someterla a los factores de corrección definidos en función de las características particulares del sitio" (Cifuentes et al., 1999, p. 11).

Capacidad de carga de manejo (CCM): La capacidad de manejo óptima se define como el mejor estado o condiciones que la administración de un área protegida debe tener para desarrollar sus actividades y alcanzar sus objetivos. Para medirla se utilizan tres tipos de variables de: personal, infraestructura y equipamiento (Cifuentes et al., 1999); teniendo en cuenta la relación entre la cantidad existente y la cantidad óptima de dichos elementos.

Capacidad de Carga Efectiva (CCE), "se emplea para estimar el límite máximo de visitas que se pueden admitir considerando las capacidades de manejo presentes en el área". (IbañezPérez, 2016, p. 46).

\subsection{Capacidad de Acogida Multicriterio (CATUM)}

A través de los años la capacidad de carga turística (CCT) ha recibido varias críticas de autores como: Watson y Kopachevsky (1996); Butler (1997); Lindberg et al, (1997); Buckley, (1999); McCool \& Lime, (2001); Vera-Rebollo \& Baños-Castiñeira, (2004); Alipour et al, (2007); López-Bonilla, \& López-Bonilla, (2008); Gutiérrez-Fernández \& Rodríguez, (2019); siendo uno de los principales inconvenientes que los turistas son únicos y rara vez se comportan de una forma homogénea, de manera que su comportamiento generan distintos tipos de impactos, por lo que resulta difícil determinar la capacidad de carga numérica del lugar visitado. No obstante, si bien la anterior afirmación es verdadera, el cálculo de la CCT en la actualidad 
sigue siendo una valiosa herramienta de planificación y gestión de la actividad del turismo y del territorio donde se realiza, por lo que, contrario a que caiga en desuso, deben proponerse formas innovadoras que permitan que se mejore el instrumento y se aumente la competitividad de los lugares receptores.

La primera diferencia entre las metodologías que se utilizan en la presente investigación radica en el uso del término acogida, como un concepto teórico que hace referencia al uso óptimo del territorio en orden a su sostenibilidad, definido por primera vez por Gómez Orea (1992) y usado posteriormente por Galacho-Jiménez \& Castaño (2008) como el grado de idoneidad o cabida que presenta el territorio para una actividad teniendo en cuenta a la vez, la medida en que el medio cubre sus requisitos locacionales y los efectos de dicha actividad sobre el medio que la rodea.

Lo anterior, significa un cambio desde la conceptualización de la metodología, ya que, al realizar el cambio del concepto principal, se logra introducir desde la etimología misma de la herramienta (CATUM) un elemento fundamental de la planeación y gestión del turismo actual, que es la sostenibilidad, que, en este caso, es el propio territorio receptor de turismo.

Teniendo en cuenta lo anterior, se realiza una modificación a la metodología de Capacidad de Carga Turística, específicamente en la forma como se calcula la capacidad de carga real (CCR), lo cual se explica en detalle en el numeral de metodología. Cabe señalar que, para el cálculo de la capacidad de carga física y la capacidad de carga efectiva, no se realizan cambios al método expuesto por Cifuentes (1990; 1992a,b; 1999).

\section{AREA DE ESTUDIO}

La Reserva Biológica Encenillo, hace parte de las zonas de protección de la Fundación Natura. Su vegetación está compuesta por un mosaico de bosques (58\%), que hacen parte de uno de los últimos relictos de bosque de Encenillo (Weinmannia tomentosa.), se encuentra un $10 \%$ de matorrales y $32 \%$ del paisaje ha sufrido procesos de potrerización. (Martínez et al, 2005). El área natural, se encuentra ubicada en la vereda La Trinidad (sector Pueblo Viejo) del municipio de Guasca, departamento de Cundinamarca - Colombia; aproximadamente a una hora de distancia del norte de Bogotá (capital de Colombia). El área tiene una extensión de 200 hectáreas aproximadamente, con alturas entre los 2800 y 3250 m.s.n.m, lo que hace que el ecosistema predominante sea Bosque Alto Andino (único para Suramérica); por su clasificación climática se encuentra en una zona muy fría, húmeda, monomodal (Marín-Santamaría, 2010).

Esta área fue reconocida como una reserva privada de la sociedad civil, abierta al público en el año 2007 y gran parte de lo que hoy está consolidado como zona de conservación funcionó durante 80 años, como un complejo minero: explotador y transformador de roca caliza, hasta que en el año 1992 cesó la minería y con ello la presión antrópica por esta actividad; desde ese momento se ha venido dando la recuperación del paisaje, en donde la naturaleza ha venido colonizando los espacios que antes eran degradados por actividades extractivas. Sin embargo, el proceso sucesional no está exento de inconvenientes, ya que en la actualidad existe la presencia de especies invasoras como el retamo espinoso (Ulex europaeus), el cual es 
abundante en varios sectores del municipio de Guasca, y detiene el proceso de restauración natural del bosque altoandino nativo (Barrera et al, 2002), además de incrementar el riesgo de incendios (Rees \& Hill, 2001, Clements et al. 2001, Beltrán-G \& Barrera-Cataño, 2014).

La reserva cuenta con cinco senderos ecoturisticos: Colibrí, Orquídeas, Thomas Van Der Hammen, Cusumbo y Mirador (Fig. 1).

Figura 1. Senderos en la reserva El Encenillo

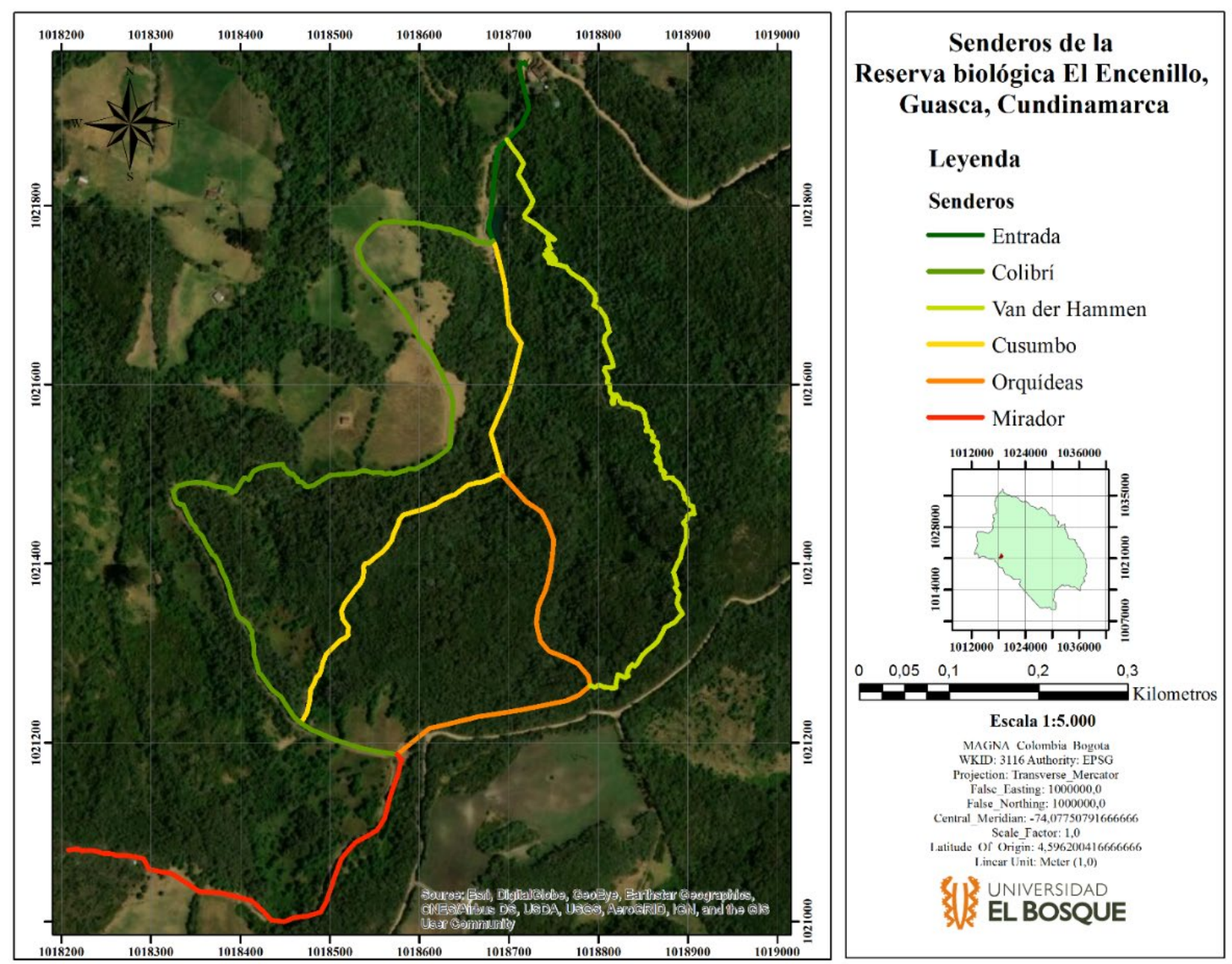

Elaboración propia, 2019.

\section{METODOLOGÍA}

La realización del presente trabajo de investigación tuvo como objetivo general comparar dos metodologías utilizadas para calcular el número máximo de visitantes que pueden ingresar diariamente a un área natural en donde se realiza turismo, de forma que se pudieran establecer las fortalezas y debilidades de cada una, por lo que se utilizaron los mismos factores de corrección en ambos casos. Para poder realizar la comparación fue necesario desarrollar 4 objetivos específicos, desarrollados en fases escalonadas:

- Establecer la línea base mediante recolección de datos en campo, 
- Calcular la Capacidad de Carga Turística (CCT),

- Calcular la Capacidad de Acogida Turística Multicriterio (CATUM),

- Realizar una comparación de resultados obtenidos con ambas metodologías.

\subsection{Establecer la línea base mediante recolección de datos en campo,}

Para realizar la recolección de las características de la reserva y poder estimar los valores para el cálculo de la CCT y la CATUM se realizaron cinco visitas a la reserva, durante el año 2019, en las que participaron 3 estudiantes de ingeniería ambiental, capacitados en técnicas de campo; cabe señalar que las dos primeras visitas fueron de diagnóstico.

La primera visita fue para hablar con los encargados de la reserva, crear alianzas de trabajo y poder establecer los intereses de la reserva en este tipo de estudio. Durante la segunda visita se realizó un recorrido general y se definieron los senderos en los que se realizaría el trabajo, dando como resultado la selección de 5 senderos. En la visita 3, 4 y 5 se recolectaron datos de cada sendero, e información para identificar y desarrollar los factores de corrección necesarios para calcular la CCT y la CATUM, como soporte tecnológico en campo se utilizó un GPS marca Garmin serie eTrex 22x, con el que se georreferenció la información, para su posterior procesamiento.

\subsection{Cálculo de la Capacidad de Carga Turística - CCT}

Para el cálculo de la Capacidad de Carga Turística de la reserva El Encenillo, se utilizaron las fórmulas y la metodología de Miguel Cifuentes (1990; 1992a,b; 1999):

- Capacidad de Carga Física = CCF

Para el cálculo se utilizaron las fórmulas propuestas por (Cifuentes et al., 1999, p15):

$\mathrm{CCF}=(\mathrm{S} / \mathrm{sp}) * \mathrm{NV}$

Donde:

$\mathrm{S}=$ superficie disponible, en metros lineales en el Sendero

$\mathrm{sp}=$ superficie usada por persona $=1 \mathrm{~m}$ de sendero

NV = número de veces que el sitio puede ser visitado por la misma persona en un día.

$\mathrm{NV}=\mathrm{Hv} / \mathrm{tv}$

Donde:

$\mathrm{Hv}=$ Horario de visita

$\mathrm{TV}=$ Tiempo necesario para visitar cada sendero

- Capacidad de Carga Real (CCR)

Se determina a partir de la capacidad de carga física multiplicándola por los factores de corrección definidos en función de las características particulares del sendero (Cifuentes et al, 1999). 
- Capacidad de carga de Manejo

La capacidad de manejo óptima se define como el mejor estado o condiciones que la administración de un área protegida debe tener para desarrollar sus actividades y alcanzar sus objetivos. Para medirla se utilizan tres tipos de variables: personal, infraestructura y equipamiento (Cifuentes et al, 1999).

\section{- Capacidad de Carga Efectiva (CCE)}

Se refiere al número máximo de visitas que se pueden permitir, en este caso en la reserva El Encenillo, y se calcula multiplicando el valor de la capacidad de carga real, por el valor de la capacidad de carga de manejo.

\subsection{Cálculo de la CATUM}

- Capacidad de Carga Física

No se realizan ajustes a la metodología de la Capacidad de Carga Turística, se calcula con las fórmulas expuestas por Cifuentes et al, (1999).

\section{- Capacidad de Carga Real (CCR)}

En esta capacidad es donde se realizan ajustes, debido a que se realiza una ponderación de los factores de corrección / variables, mediante el uso de la metodología de análisis multicriterio propuesta por Thomas Saaty (1980), denominada como Proceso Analítico Jerárquico (Analytic Hierarchy Process - AHP).

El método AHP, es un método que emplea la construcción de un modelo jerárquico, y posteriormente evalúa y entrega una ordenación de las alternativas de solución desde la mejor hasta la peor. Se fundamenta en las comparaciones pareadas, utilizando una gradación con valores de 1 a 9 para calificar las preferencias relativas de los dos elementos evaluados, a la cual también se le denomina como escala Saaty.

La ventaja del AHP consiste en que permite incorporar aspectos cualitativos que suelen quedarse por fuera de los análisis debido a su complejidad para ser medidos, pero que pueden ser relevantes para algunos actores involucrados en la toma de decisión (Gutiérrez-Fernández, 2011).

El procedimiento matemático para obtener una solución fue el del promedio por filas de los elementos normalizados de cada columna de la matriz y la normalización de la suma de los elementos de cada fila. Cuando se dispone de una escala, las prioridades relativas de los elementos que cuelgan de un nodo son conocidas directamente. En este caso, la matriz recíproca de comparaciones pareadas, $\mathrm{W}=(\mathrm{wi} / \mathrm{wj})$, queda como: 


$$
\left(\begin{array}{cccc}
\mathrm{w}_{1} / \mathrm{w}_{1} & \mathrm{w}_{1} / \mathrm{w}_{2} & \ldots & \mathrm{w}_{1} / \mathrm{w}_{\mathrm{n}} \\
\mathrm{w}_{2} / \mathrm{w}_{1} & \mathrm{w}_{2} / \mathrm{w}_{2} & \ldots & \mathrm{w}_{2} / \mathrm{w}_{\mathrm{n}} \\
\ldots & \ldots & \ldots & \ldots \\
\mathrm{w}_{\mathrm{n}} / \mathrm{w}_{1} & \mathrm{w}_{\mathrm{n}} / \mathrm{w}_{2} & \ldots & \mathrm{w}_{\mathrm{n}} / \mathrm{w}_{\mathrm{n}}
\end{array}\right)
$$

En este caso, la matriz $\mathrm{W}$ anterior tiene rango uno, con lo que el problema del autovector se reduce a $W w=n w$, con $\sum j w j=1$. Una forma sencilla de obtener el valor de $\lambda$ max si se conoce el valor exacto de w (o estimación) en forma normalizada, es sumar las columnas de $A$ y multiplicar el vector resultante por el vector de prioridades w. En general, utilizando el teorema de PerronFrobenius, se puede probar que $\lambda \max \geq n$ para el método de AHP (Saaty, 1980; Moreno-Jiménez, 2002)

Para disminuir la subjetividad de la ponderación se utilizó la consulta a terceros, empleando la metodología denominada 3s (metodología de validación en 3 niveles) propuesta por Cloquell (2003), cuyas características fundamentales se describen con mayor detalle a continuación:

- Nivel 1: SUI Validatio

La autovalidación debe ser realizada por el propio equipo de redacción del estudio. Su propósito es: realizar una reflexión interna de lo propuesto.

- Nivel 2: SCIENCIATIS Validatio

La validación científica pretende dotar de rigor y objetividad a los que han sido diseñados por el equipo redactor mediante la integración de los juicios de expertos independientes.

- Nivel 3: SOCIETATIS Validatio

Este tipo de validación social pretende incorporar la participación pública, elemento muy importante para la gestión del turismo y la incorporación de las poblaciones locales, lo que permite derribar algunas de las barreras que supone la gestión adecuada de las áreas naturales y que además su realización dota al proceso de una mayor transparencia y de una mayor posibilidad de éxito (OMT, 2005).

Es importante destacar que la metodología escogida ha sido utilizada en estudios como: Cloquell-Ballester et al, 2006; Gutiérrez-Fernández et al, 2012; Barrera-Tolosa, 2012; y por Gutiérrez-Fernández \& Rodríguez, 2019.

Ahora bien, los valores de prioridad obtenidos con la metodología AHP para garantizar la calidad de la decisión final, deben ser sometidos a la consistencia de los juicios que muestran los tomadores de decisiones en el transcurso de la serie de comparaciones pareadas. Se debe considerar que la obtención de consistencias perfectas es muy difícil de logar, sin 
embargo, el AHP tiene un método para medir el grado de consistencia entre las opiniones pareadas que proporciona el decisor. Si se consigue una razón de consistencia menor 0.10 se considera aceptable, de manera que se puede continuar con el proceso; de no ser así, quien toma las decisiones debe reconsiderar y modificar sus juicios sobre las comparaciones pareadas antes de continuar con el calculo. (Berumen \& Redondo, 2007).

La fórmula matemática de este cálculo es $\mathbf{A} n x n$ es consistente si: $\mathrm{a}_{\mathrm{ij}} \mathrm{a}_{\mathrm{jk}}=i, j, k=1,2$, $\ldots, n$. Está propiedad requiere que todas las columnas (y renglones) de $\mathbf{A}$ sean linealmente dependientes.

El Indice de Consistencia de A se calcula entonces como:

$$
I C=\frac{n_{\max }-n}{n-1}
$$

Como resultado final se calcula la Razón de Consistencia ( $\mathbf{R C}$ ) como el cociente entre el Índice de Consistencia de $\mathbf{A}$ y Índice Aleatorio de consistencia

$$
R C=\frac{I C}{I A}
$$

IA es el Índice Aleatorio de consistencia de A, es el índice de consistencia de una matriz de comparaciones pareadas generada en forma aleatoria. Se puede mostrar que IA depende del número de elementos que se comparan y asume los siguientes valores, propuestos por Saaty (1980).

- Capacidad de carga de Manejo

No tiene ajustes, se calcula con las fórmulas expuestas por Cifuentes et al (1999).

- Capacidad de Carga Efectiva (CCE)

No tiene ajustes, se calcula con las fórmulas expuestas por Cifuentes et al (1999).

\subsection{Comparación de resultados}

Se realiza una comparación de los resultados obtenidos con ambas metodologías con el fin de realizar un análisis de los mismos, de las ventajas y las dificultades que se encuentran en cada una, lo cual servirá para futuras investigaciones con el objetivo de robustecer la forma como se calcula el número máximo de visitantes que puede recibir un área natural con uso turístico y como se realiza la planificación del turismo a partir de estos datos. 


\section{RESULTADOS}

\subsection{Capacidad de Carga Turística - CCT}

A continuación, se presentan en primer lugar los resultados de la capacidad de carga turística:

\subsubsection{Capacidad de carga Física}

El acceso a la reserva se realiza por un único sendero (Sendero Entrada) el cual carece de una importancia turística significativa, ya que está altamente intervenido, por lo que no se realizó el cálculo para el mismo, entendiendo que el recorrido empieza cuando llegan a los senderos Colibrí, Van der Hammen o Cusumbo (ver figura 1).

La capacidad de carga física se calculó para los 5 senderos de la reserva que puede escoger el visitante, siendo estos: Colibrí, Van der Hammen, Cusumbo, Orquídeas y Mirador, (ver fig. 1 y tabla 1 ).

Tabla 1. Capacidad de carga física de la Reserva El Encenillo

\begin{tabular}{|c|c|c|c|c|c|c|}
\hline SENDERO & $\begin{array}{c}\text { LARGO DEL } \\
\text { SENDERO } \\
(\mathbf{m})\end{array}$ & $\begin{array}{c}\text { ANCHO } \\
\text { PROMEDIO } \\
\text { SENDERO (m) }\end{array}$ & $\begin{array}{c}\text { HORAS } \\
\text { ABIERTO AL } \\
\text { PÚBLICO (Hv) }\end{array}$ & $\begin{array}{c}\text { TIEMPO NECESARIO } \\
\text { PARA VISITAR CADA } \\
\text { SENDERO (Tv) }\end{array}$ & $\begin{array}{c}\text { Hv / } \\
\text { tv }\end{array}$ & CCF \\
\hline Colibrí & 1221,09 & 1,43 & 540 & 240 & 2,25 & $\mathbf{2 7 4 7 , 4 5 2 5}$ \\
\hline Orquídeas & 523,75 & 0,78 & 540 & 60 & 9 & $\mathbf{4 7 1 3 , 7 5}$ \\
\hline Mirador & 516,97 & 1,51 & 540 & 80 & 6,8 & $\mathbf{3 4 9 0}$ \\
\hline Cusumbo & 677,62 & 1,16 & 540 & 90 & 6 & $\mathbf{4 0 6 5 , 7 2}$ \\
\hline $\begin{array}{c}\text { Van der } \\
\text { Hammen }\end{array}$ & 916,87 & 0,86 & 540 & 90 & 6 & $\mathbf{5 5 0 1 , 2 2}$ \\
\hline
\end{tabular}

Elaboración propia, 2020.

Para los senderos Colibrí, Orquídeas, Mirador, y Van der Hammen, el tiempo necesario para visitar el sendero se calculó doble, teniendo en cuenta que el visitante puede regresar por el mismo. El único que no tiene un reforzamiento en el impacto es Cusumbo, ya que las fuertes pendientes del mismo hacen que el turista escoja otro sendero para su regreso.

\subsubsection{Capacidad de Carga Real}

Para poder definir los factores de corrección (variables) se realizó una revisión de los usados con mayor frecuencia en estudios similares y se descartaron aquellos que por las condiciones naturales, de acceso y propias de los senderos, no eran aplicables o tenían una menor relevancia.

De acuerdo a lo anterior se establecieron 3 categorías (físicas, ecológicas y sociales) y ocho (8) variables/ factores de corrección en total (ver Tabla 2). 
Tabla 2. Factores de corrección para el cálculo de la capacidad de carga real de la Reserva El Encenillo

\begin{tabular}{|c|c|c|}
\hline CATEGORIA FÍSICA & CATEGORIA ECOLÓGICA & CATEGORIA SOCIAL \\
\hline FC Erodabilidad & FC Fauna & FC social \\
\hline FC Accesibilidad & FC Flora & \\
\hline FC Anegamiento & FC retamo espinoso & \\
\hline FC Precipitación & & \\
\hline
\end{tabular}

Elaboración propia, 2020.

\subsubsection{Categoría Física}

Para obtener los factores de corrección de esta categoría, fue necesario establecer las curvas de nivel, de forma que se tuvieran los valores para el cálculo de las pendientes (ver fig. 2).

Figura 2. Curvas de nivel de la Reserva El Encenillo

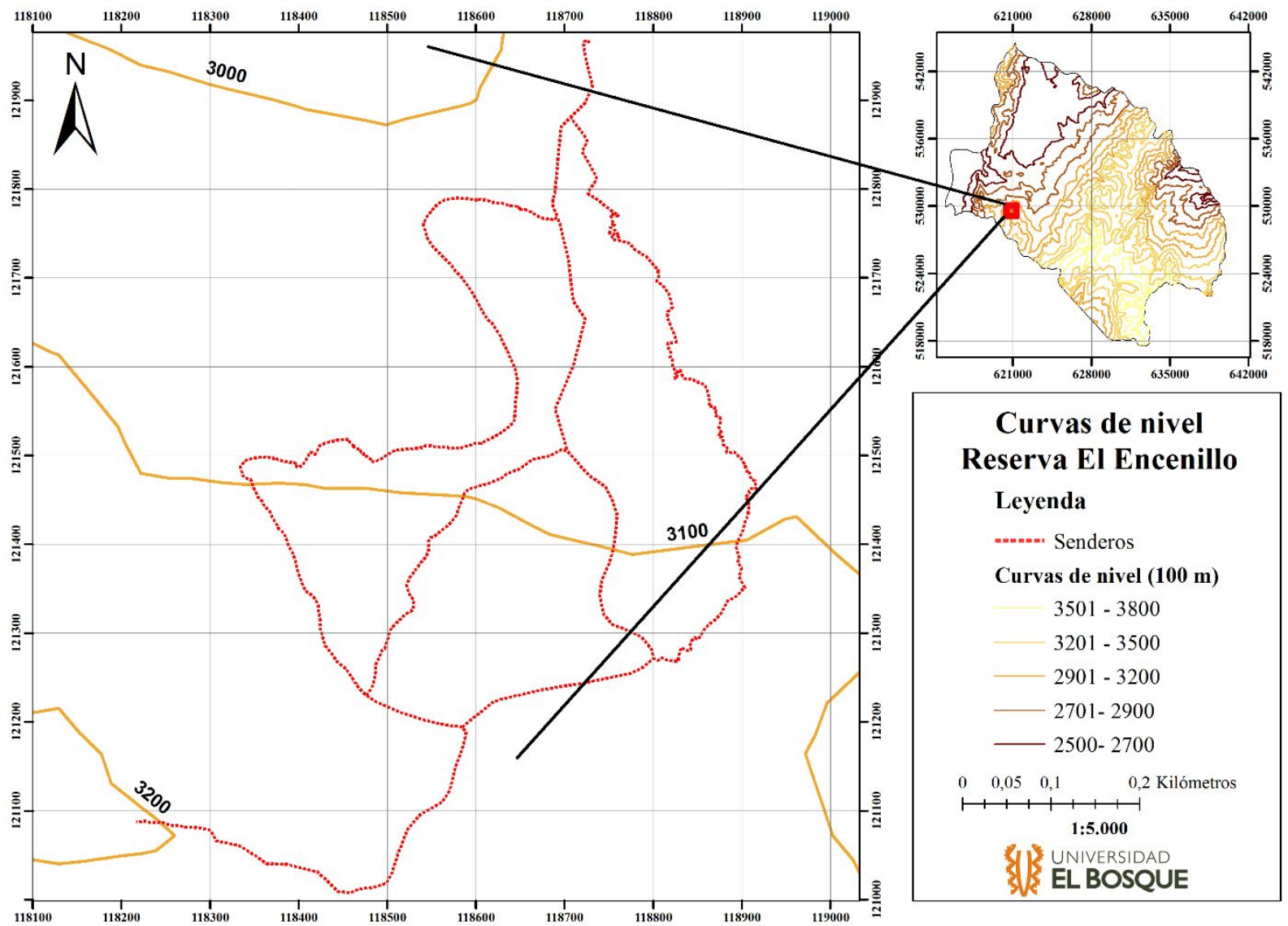

Elaboración propia, 2020.

A continuación, se muestran los resultados obtenidos para la Capacidad de Carga Real con los factores de corrección de la categoría física. 
Tabla 3. Cálculo de la CCR con factores de corrección de la categoría física

\begin{tabular}{|c|c|c|c|c|c|}
\hline SENDERO & $\begin{array}{c}\text { FC } \\
\text { ERODABILIDAD }\end{array}$ & $\begin{array}{c}\text { FC } \\
\text { ACCESIBILIDAD }\end{array}$ & $\begin{array}{c}\text { FC } \\
\text { ANEGAMIENTO }\end{array}$ & $\begin{array}{c}\text { FC } \\
\text { PRECIPITACIÓN }\end{array}$ & $\begin{array}{c}\text { CCR CATEGORÍA } \\
\text { FísICA }\end{array}$ \\
\hline COLIBRÍ & 0,899 & 1 & 0,989 & 0,452 & $\mathbf{1 1 0 4}$ \\
\hline ORQUÍDEAS & 0,973 & 0,989 & 0,997 & 0,452 & $\mathbf{2 0 4 4}$ \\
\hline MIRADOR & 0,971 & 0,963 & 0,999 & 0,452 & $\mathbf{1 4 7 4}$ \\
\hline CUSUMBO & 0,945 & 0,991 & 0,993 & 0,452 & $\mathbf{1 7 0 9}$ \\
\hline $\begin{array}{c}\text { VAN DER } \\
\text { HAMMEN }\end{array}$ & 0,986 & 1 & 0,991 & 0,452 & $\mathbf{2 4 3 0}$ \\
\hline
\end{tabular}

Elaboración propia, 2020.

\subsubsection{Categoría ecológica}

\subsection{Factor de corrección fauna}

Para calcular el factor de corrección de fauna, se tuvieron en cuenta dos especies cusumbo (Nasua nasua) y zorro (Cerdocyon thousque), las cuales están en la categoría de preocupación menor de la Unión Internacional para la Conservación de la Naturaleza. Para su escogencia se tuvieron además del grado de amenaza, tres variables: desplazamiento por tránsito de visitantes, presencia de individuos en el sendero y grado de amenaza de la especie.

Tabla 4. Calificación para las especies de fauna, avistadas en los senderos.

\begin{tabular}{|c|c|c|c|c|}
\hline \multirow[b]{2}{*}{ VALOR } & \multirow[b]{2}{*}{$\begin{array}{l}\text { ESCALA DE } \\
\text { CALIFICACIÓN }\end{array}$} & \multicolumn{3}{|c|}{ DESCRIPCIÓN CALIFICACIÓN PARA } \\
\hline & & $\begin{array}{l}\text { DESPLAZAMIENTO POR } \\
\text { TRÁNSITO DE VISITANTES }\end{array}$ & $\begin{array}{l}\text { PRESENCIA DE } \\
\text { INDIVIDUOS EN EL } \\
\text { SENDERO }\end{array}$ & $\begin{array}{l}\text { GRADO DE AMENAZA } \\
\text { DE LA ESPECIE }\end{array}$ \\
\hline 1 & Bajo & $\begin{array}{l}\text { La especie no se ve afectada } \\
\text { por el tránsito de turistas }\end{array}$ & $\begin{array}{l}\text { Casi nunca hay } \\
\text { avistamientos o } \\
\text { rastros de la especie } \\
\text { en el lugar de estudio }\end{array}$ & $\begin{array}{l}\text { La especie se encuentra } \\
\text { con un bajo nivel de } \\
\text { amenaza y su población } \\
\text { es estable en el área de } \\
\text { estudio. }\end{array}$ \\
\hline 2 & Medio & $\begin{array}{c}\text { La especie desaparece } \\
\text { esporádicamente del área, } \\
\text { ante la presencia de turistas, } \\
\text { pero regresa a su lugar de } \\
\text { hábitat. }\end{array}$ & $\begin{array}{l}\text { Hay avistamientos o } \\
\text { rastros esporádicos } \\
\text { de la especie en el } \\
\text { lugar de estudio }\end{array}$ & $\begin{array}{l}\text { La especie se ve } \\
\text { amenazada por factores } \\
\text { antrópicos o naturales, } \\
\text { sin embargo, su } \\
\text { población se encuentra } \\
\text { estable. }\end{array}$ \\
\hline 3 & Alto & $\begin{array}{l}\text { La especie desaparece del } \\
\text { área de hábitat ante la } \\
\text { presencia de turistas y le } \\
\text { cuesta regresar a dicho sitio. }\end{array}$ & $\begin{array}{l}\text { Hay constantes } \\
\text { avistamientos o } \\
\text { rastros de la especie } \\
\text { en el lugar de estudio }\end{array}$ & $\begin{array}{l}\text { La especie se ve } \\
\text { amenazada por varios } \\
\text { factores antrópicos y } \\
\text { naturales, afectando } \\
\text { la estabilidad de su } \\
\text { población }\end{array}$ \\
\hline
\end{tabular}

Fuente: Gomez et al., 2016.

Para la primera variable, se determinó que ambas especies desaparecen esporádicamente del área ante la presencia de turistas, pero regresan a su lugar de hábitat, por lo que se les dio un valor de 2 , es decir, medio. 
Para la segunda variable, se asignó un valor de 3, alto, pues hay constantes avistamientos o rastros de la especie en el lugar de estudio.

Para la tercera variable, se determinó que ambas especies se encuentran en una escala baja, con un valor de 1 porque, de acuerdo con lo afirmado por los guías de la reserva, se encuentran con un bajo nivel de amenaza y su población es estable en el área de estudio.

Tabla 5. Calificación de las variables para cada especie

\begin{tabular}{|c|c|c|c|c|c|}
\hline NOMBRE COMÚN & NOMBRE CIENTÍfICO & VARIABLE 1 & VARIABLE 2 & VARIABLE 3 & TOTAL \\
\hline CUSUMBO & Nasuella olivacea & 2 & 3 & 1 & 6 \\
\hline ZORRO & Cerdocyon thous & 2 & 3 & 1 & 6 \\
\hline
\end{tabular}

Elaboración propia, 2020.

La calificación obtenida para ambas especies, como se muestra en la tabla No.5, es de un valor de 6 , por lo tanto, se considera que es una especie relevante a tener en cuenta en el cálculo de la capacidad de carga.

Para encontrar el sub factor de corrección del cusumbo, se tomó un periodo de gestación de 74 días al año, al cual se le sumó el tiempo que requieren las crías para poder reincorporarse al grupo, llegando a un total de 116 días (Yupanqui, 2005). Por lo tanto, el sub-factor de corrección del Cusumbo es 0,68; valor obtenido de aplicar la siguiente formula )

$$
\text { factor de corrección }=1-\frac{\text { factor limitante }}{\text { magnitud total }} \text {. }
$$

Para encontrar el sub factor de corrección del zorro, se estableció que el periodo de gestación es de 56 días al año y una actividad predominantemente nocturna (Medel, \& Jaksic, 1988). Por lo tanto, el sub-factor de corrección para esta especie es 0,8465 .

Para el factor de corrección de fauna, se tuvo en cuenta la presencia / ausencia de las especies en cada uno de los senderos, por lo que, para Orquídeas, Cusumbo y Van der Hammen el cálculo se realiza con la suma del subfactor de ambas especies y se divide por 2 . En el caso de colibrí y mirador sólo se tiene en cuenta el subfactor del zorro, ya que no hay presencia de cusumbo (ver tabla No. 6).

Tabla 6. Cálculo de factor de corrección fauna

\begin{tabular}{|c|c|c|}
\hline SENDERO & PRESENCIA / AUSENCIA DE ESPECIES & FC FAUNA \\
\hline Colibrí & Zorro (Cerdocyon thous) & 0.8465 \\
\hline Orquídeas & Zorro (Cerdocyon thous) y Cusumbo (Nasuella olivacea) & 0.7643 \\
\hline Mirador & Zorro (cerdocyon thous) & 0.8465 \\
\hline Cusumbo & Zorro (Cerdocyon thous) y Cusumbo (Nasuella olivacea) & 0.7643 \\
\hline Van der Hammen & Zorro (Cerdocyon thous) y Cusumbo (Nasuella olivacea) & 0.7643 \\
\hline
\end{tabular}

Elaboración propia, 2020. 


\subsection{Factor de corrección flora}

El factor de corrección flora se realiza teniendo en cuenta dos géneros que fueron: el primero Weinmania, específicamente la especie W. tomentosa (Encenillo) y el Espeletia (frailejon) dentro del cual se identificaron dos especies (Espeletia grandiflora y Espeletia argentea).

La especie Weinmania tomentosa se escoge dado que, si bien no sufre una afectación directa por el turismo, sí se ven afectadas sus semillas, al ser sometida a pisoteo la hojarasca, lo que impide que nazcan nuevas plántulas y que pueda verse afectado el proceso sucesional y la regeneración natural.

El género Espeletia (especies E. grandiflora y E. argentea), se escoge ya que sus especies son muy sensibles, poco resilientes y de muy lento crecimiento; adicionalmente durante los recorridos de campo se observaron individuos afectados por el paso del turista.

\section{Figura 3. Flora nativa amenazada en El Encenillo}

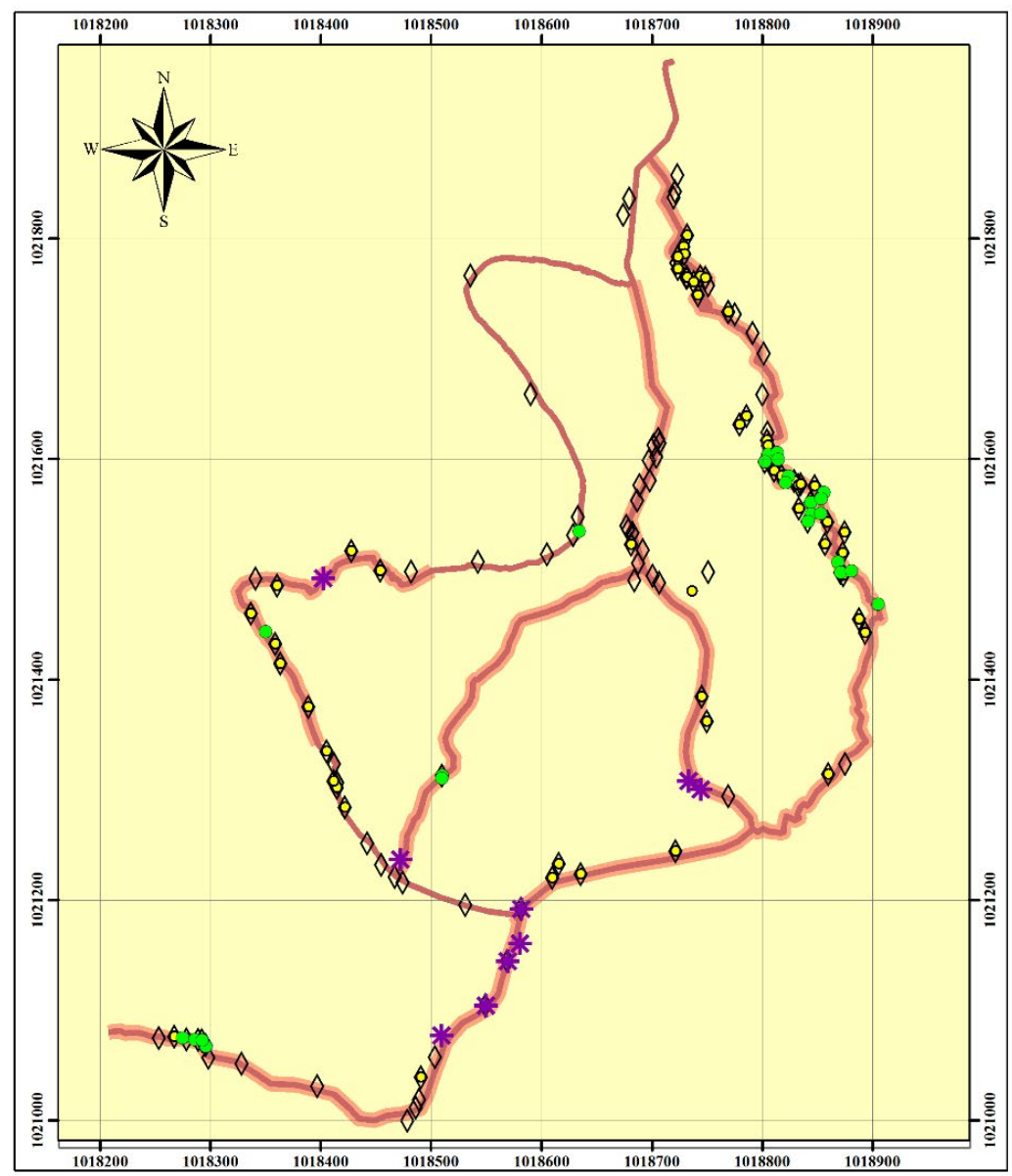

Flora nativa amenazada por las especies invasoras (Ulex Europeus) y (Rubus Ulmifolius)

Leyenda

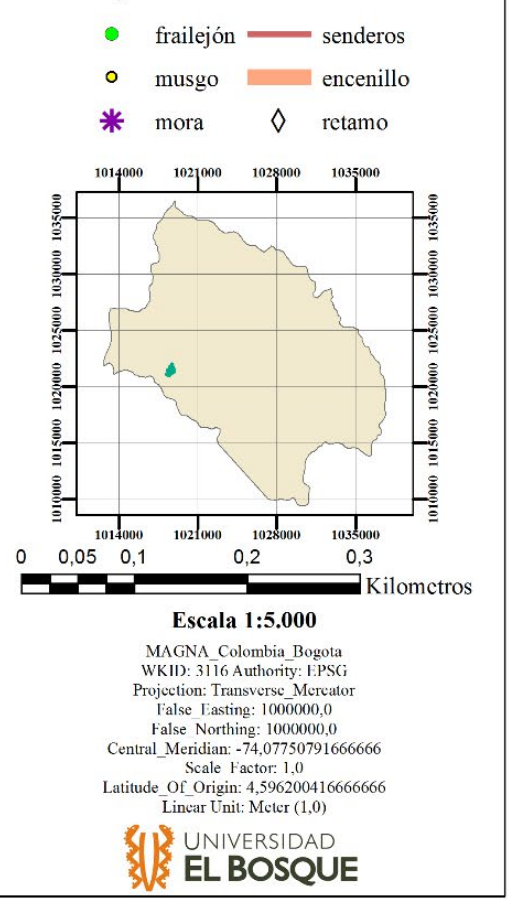

Elaboración propia, 2020. 
Tabla 7. Longitud de coberturas vegetales en los senderos

\begin{tabular}{|c|c|c|c|c|c|}
\hline SENDERO & $\begin{array}{c}\text { ESPECIE(S) } \\
\text { REPRESENTATIVA(S) }\end{array}$ & NOMBRE CIENTíFICO & $\begin{array}{c}\text { LONGITUD TOTAL } \\
\text { DEL SENDERO CON } \\
\text { PRESENCIA DE LA(s) } \\
\text { ESPECIE(s) (m) }\end{array}$ & $\begin{array}{c}\text { LARGO DEL } \\
\text { SENDERO (m) }\end{array}$ & $\begin{array}{c}\text { FACTOR DE } \\
\text { CORRECCIÓN }\end{array}$ \\
\hline COLIBRÍ & - & - & - & 1221,09 & 1,0000 \\
\hline ORQUÍDEAS & Encenillo & Weinmania tomentosa & 285,13 & 523,75 & 0,4556 \\
\hline MIRADOR & $\begin{array}{c}\text { Frailejón } \\
\text { Frailejón }\end{array}$ & $\begin{array}{c}\text { Espeletia grandiflora. } \\
\text { Espeletia argentea }\end{array}$ & 99,33 & 516,97 & 0,8079 \\
\hline CUSUMBO & $\begin{array}{c}\text { Encenillo } \\
\text { Frailejón }\end{array}$ & $\begin{array}{c}\text { Weinmania tomentosa } \\
\text { Espeletia grandiflora }\end{array}$ & 206,99 & 677,62 & 0,6945 \\
\hline $\begin{array}{c}\text { VAN DER } \\
\text { HAMMEN }\end{array}$ & $\begin{array}{c}\text { Encenillo } \\
\text { Frailejón }\end{array}$ & $\begin{array}{c}\text { Weinmania tomentosa } \\
\text { Espeletia argentea }\end{array}$ & 59,11 & 916,87 & 0,9355 \\
\hline
\end{tabular}

Elaboración propia, 2020.

Figura 4. Fauna y flora representativas en El Encenillo

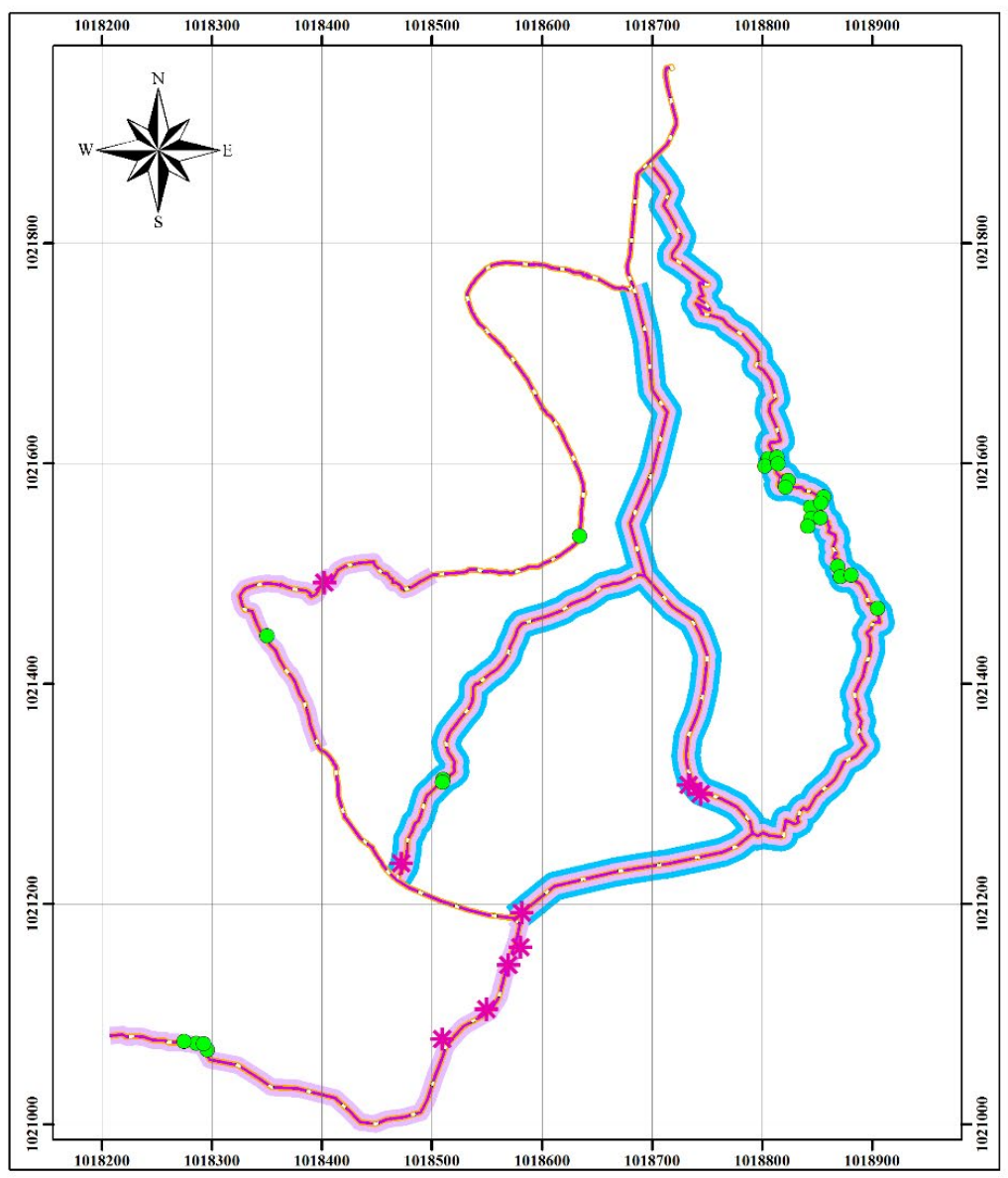

\section{Presencia de fauna y flora representativas en la Reserva biológica El Encenillo}

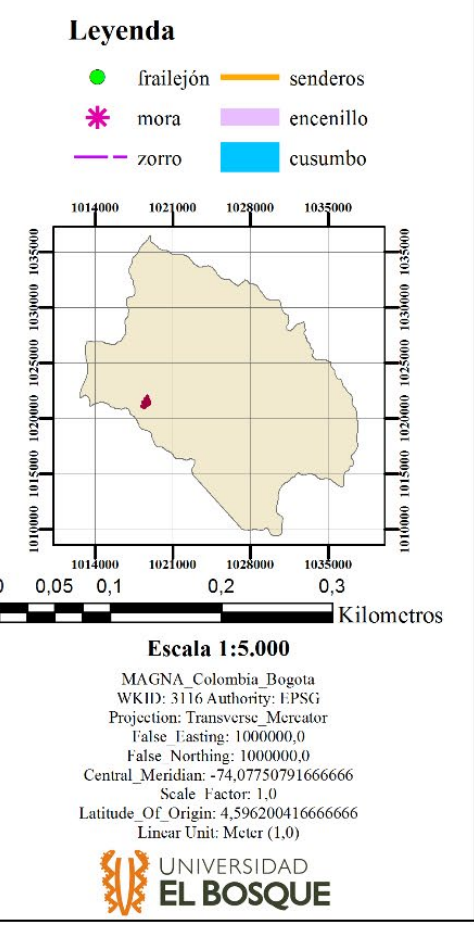

Elaboración propia, 2020. 


\subsection{Factor de corrección especie invasora (Ulex europaeus)}

Por último, se calcula como factor de corrección el retamo espinoso que es una especie invasora originaria de la costa occidental de Europa y norte de África (Clements et al., 2001) y es considerada por varios estudios como una de las 100 especies invasoras más agresivas del mundo (Lowe et al., 2000; Aguilar, 2010; Baptiste et al., 2010; Kaal et al., 2012; OcampoZuleta \& Solorza-Bejarano, 2017), presenta un rápido crecimiento y alta capacidad de invasión (Moore., 2006; Udo et al., 2016), con alta producción y longevidad de semillas que adicionalmente son muy fácilmente transportadas en la ropa de los caminantes, así como tener un alto porcentaje de germinación, y presentar características adversas para la conservación del ecosistema de bosque alto andino, como la de ser ignífuga, lo que pone en riesgo la reserva. En la figura 5, se puede observar donde se encuentran los individuos de esta planta, a lo largo de los senderos.

Figura 5. Presencia de especie invasora (Ulex europaeus) en El Encenillo
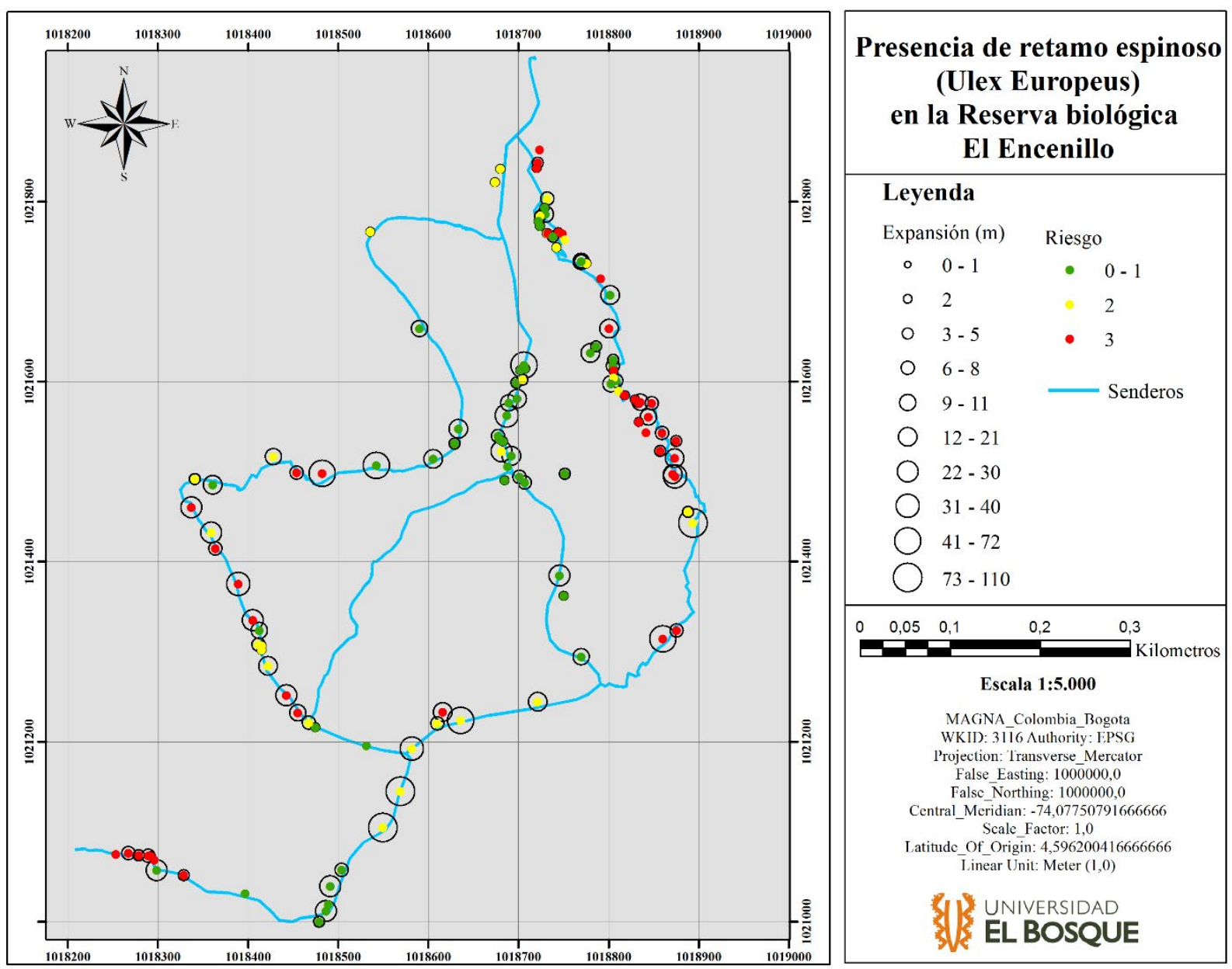

Elaboración propia, 2020.

Con los tres factores: fauna, flora y especie invasora (Ulex europaeus), se calcula la Capacidad de Carga Real en la categoría ecológica (Ver tabla No. 8). 
Tabla 8. Capacidad de carga real con factores de corrección categoría ecológica

\begin{tabular}{|c|c|c|c|c|}
\hline SENDERO & FC FAUNA & FC FLORA & $\begin{array}{c}\text { FC ESPECIE } \\
\text { INVASORA (Ulex } \\
\text { europaeus) }\end{array}$ & $\begin{array}{c}\text { CCR } \\
\text { CATEGORÍA ECOLÓGICA }\end{array}$ \\
\hline Colibrí & 0.8465 & 1,0000 & 0,3261 & $\mathbf{3 0 5}$ \\
\hline Orquídeas & 0.7643 & 0,4556 & 0,2740 & $\mathbf{1 9 5}$ \\
\hline Mirador & 0.8465 & 0,8079 & 0,8646 & $\mathbf{8 6 9}$ \\
\hline Cusumbo & 0.7643 & 0,6945 & 0,2873 & $\mathbf{2 6 0}$ \\
\hline Van der Hammen & 0.7643 & 0,9355 & 0,4403 & $\mathbf{7 5 5}$ \\
\hline
\end{tabular}

Elaboración propia, 2020.

\subsubsection{Categoría social}

Para determinar el factor de corrección de la categoría social se determinó el área del grupo, la longitud necesaria para el desplazamiento del mismo, el número de grupos que pueden estar simultáneamente en el sendero, el número de personas que pueden estar simultáneamente en el sendero y la magnitud limitante.

Tabla 9. Capacidad de carga real con factor de corrección categoría social

\begin{tabular}{|c|c|c|}
\hline SENDERO & FC SOCIAL & $\begin{array}{c}\text { CCR } \\
\text { CATEGORÍA SOCIAL }\end{array}$ \\
\hline Colibrí & 0,0364 & 11,1020 \\
\hline Orquídeas & 0,0364 & 7,0980 \\
\hline Mirador & 0,0364 & 31,6316 \\
\hline Cusumbo & 0,0364 & 9,4640 \\
\hline Van der Hammen & 0,0364 & 27,4820 \\
\hline
\end{tabular}

Elaboración propia, 2020.

Cabe señalar que con el cálculo de la capacidad de carga real - categoría social finaliza el computo de ésta.

\section{Capacidad de Carga Efectiva}

A continuación, se presentan los resultados de la capacidad de carga efectiva, para lo cual se calculó la capacidad de manejo de la Reserva el Encenillo.

\subsubsection{Capacidad de manejo}

Por último, la capacidad de manejo se estimó a partir del estado de tres variables: infraestructura, equipamiento y personal. Con ello, se obtuvo la Tabla No. 10. 
Tabla 10. Resultados capacidad de manejo

\begin{tabular}{|c|c|c|}
\hline VARIABLE GENERAL & VALOR & CAPACIDAD DE MANEJO (\%) \\
\hline Infraestructura & 2 & $70 \%$ \\
\hline Equipamiento & 2 & $55 \%$ \\
\hline Personal & 2 & $63 \%$ \\
\hline \multicolumn{2}{|c|}{ Capacidad de manejo de la reserva } & $63 \%$ \\
\hline
\end{tabular}

Elaboración propia, 2020.

Si bien la reserva tiene como finalidad la conservación, es necesario que mejoré la infraestructura para la prestación del servicio turístico, al igual que el equipamiento con el que se cuenta, y el personal, en cuanto a número y competencias contratado para que guie al visitante.

Lo anterior, no solo permitirá mejorar la calidad de la experiencia del visitante, sino aumentar la capacidad de carga turística que puede permitirse dentro de la reserva.

Con el valor de la capacidad de manejo, se procede a calcular efectivamente cuantos son los turistas que pueden ingresar a la Reserva El Encenillo de acuerdo con la metodología de CCT.

Tabla 11. Cálculo capacidad de carga efectiva metodología CCT

\begin{tabular}{|c|c|c|c|}
\hline SENDERO & $\begin{array}{c}\text { PORCENTAJE DE } \\
\text { CAPACIDAD DE MANEJO }\end{array}$ & $\begin{array}{c}\text { CAPACIDAD DE CARGA } \\
\text { REAL }\end{array}$ & $\begin{array}{c}\text { CAPACIDAD DE CARGA } \\
\text { EFECTIVA- } \\
\text { CCT }\end{array}$ \\
\hline Colibrí & $63 \%$ & 11,1020 & $\mathbf{7}$ \\
\hline Orquídeas & $63 \%$ & 7,0980 & $\mathbf{4}$ \\
\hline Mirador & $63 \%$ & 31,6316 & $\mathbf{2 0}$ \\
\hline Cusumbo & $63 \%$ & 9,4640 & $\mathbf{6}$ \\
\hline Van der Hammen & $63 \%$ & 27,4820 & $\mathbf{1 7}$ \\
\hline
\end{tabular}

Elaboración propia, 2020.

La capacidad de carga final de acuerdo con la metodología CCT, arroja un número inferior de visitantes/día que pueden ingresar a los senderos: Colibrí, Orquídeas y Cusumbo inferiores a las personas que se manejan en la actualidad por cada grupo (la reserva maneja un número de 17 personas, por grupo).

Lo anterior, puede ser inviable económicamente y presionar aún más la capacidad de carga de manejo, medida en infraestructura, equipamiento y personal, siendo las dos últimas variables las que se pueden ver más afectadas, ya que, al reducir el número de visitantes por grupo, es previsible que se requieran mayores valores en equipos y funcionarios disponibles para realizar la guianza. 


\subsection{Capacidad de Acogida Turística Multicriterio (CATUM)}

Como se explicó en el apartado de metodología, la única capacidad de carga que se ajusta es la real para el cálculo de la Capacidad de Acogida Turística Multicriterio, por lo que a continuación se muestran de forma desagregada los resultados en esta categoría de acuerdo a la metodología CATUM.

\subsubsection{Capacidad de carga Física}

Los resultados son iguales que con la capacidad de carga turística, ya que la metodología en este ítem no sufre variaciones con respecto a la de Cifuentes (1990; 1992a,b; 1999) (ver tabla 1).

\subsubsection{Capacidad de Carga Real (CCR):}

En esta capacidad es donde se realizan ajustes, dado que se lleva a cabo una ponderación, de los factores de corrección, utilizando la metodología 3's o en 3 niveles, como se muestra a continuación:

\subsubsection{Ponderación de los factores de corrección por categoría}

\subsection{Ponderación de los factores de corrección - categoría física}

En este apartado se muestran los resultados de la ponderación realizada por el grupo de investigación para los factores de corrección pertenecientes a la categoría física:

Tabla 12. Ponderación nivel 1. Sui Validatio de los factores de corrección de la categoría física

\begin{tabular}{|c|c|}
\hline FACTOR DE CORRECCIÓN & PORCENTAJE \\
\hline FC Erodabilidad & $25 \%$ \\
\hline FC Accesibilidad & $25 \%$ \\
\hline FC Anegamiento & $25 \%$ \\
\hline FC Precipitación & $25 \%$ \\
\hline Razón de consistencia & 0,00 \\
\hline
\end{tabular}

Elaboración propia, 2020.

Para la ponderación en el nivel científico (Scientatis), participaron dos expertos en temas de turismo con nivel de maestría y con más de 5 años de experiencia laboral. Para el desarrollo de su ponderación se utilizó el software Expert Choice Comparion ${ }^{\circledR}$, que es un programa informático basado en el modelo AHP para la priorización y optimización de recursos, y por su sencillez permite que los participantes vayan viendo la consistencia de sus respuestas a medida que van avanzando en el proceso de priorización (este software se utilizó también con los participantes del nivel social - societatis), es necesario señalar que las razones de consistencia de todos los participantes fueron menores a 0.10 , por lo que puede decirse que son 
Cálculo de la capacidad de carga y capacidad de acogida turística multicriterio para la reserva biológica El Encenillo, Guasca, Cundinamarca, Colombia

consistentes y es posible continuar con el proceso de cálculo de la Capacidad de Carga Real, ya que los decisores realizaron juicios sólidos. Los resultados obtenidos fueron los siguientes:

Tabla 13. Ponderación nivel Scientatis Validatio de los factores de corrección de la categoría física

\begin{tabular}{|c|c|c|c|}
\hline FACTORES DE CORRECCIÓN & $\begin{array}{c}\text { EXPERTO } \\
\mathbf{1}\end{array}$ & $\begin{array}{c}\text { EXPERTO } \\
\mathbf{2}\end{array}$ & PROMEDIO \\
\hline FC Erodabilidad & $43,21 \%$ & $32,50 \%$ & $37,86 \%$ \\
\hline FC Accesibilidad & $19,52 \%$ & $24,17 \%$ & $21,85 \%$ \\
\hline FC Anegamiento & $19,52 \%$ & $24,17 \%$ & $21,85 \%$ \\
\hline FC Precipitación & $17,74 \%$ & $19,17 \%$ & $18,45 \%$ \\
\hline Razón de consistencia & 0,01 & 0,06 & \\
\hline
\end{tabular}

Elaboración propia, 2020.

Para la ponderación en el nivel social (societatis), participaron cinco personas, con formación en temas ambientales (pregrado) y que son visitantes de este tipo de reservas y tienen un alto interés por el turismo sostenible. Para el desarrollo de su ponderación al igual que con el nivel científico (Scientatis), se utilizó el software Expert Choice Comparion ${ }^{\circledR}$, los resultados obtenidos fueron:

Tabla 14. Ponderación nivel Societatis Validatio de los factores de corrección de la categoría física

\begin{tabular}{|c|c|c|c|c|c|c|}
\hline $\begin{array}{c}\text { FACTORES DE } \\
\text { CORRECCIÓN }\end{array}$ & $\begin{array}{c}\text { VISITANTE } \\
1\end{array}$ & $\begin{array}{c}\text { VISITANTE } \\
2\end{array}$ & $\begin{array}{c}\text { VISITANTE } \\
3\end{array}$ & $\begin{array}{c}\text { VISITANTE } \\
4\end{array}$ & $\begin{array}{c}\text { VISITANTE } \\
5\end{array}$ & PROMEDIO \\
\hline FC Erodabilidad & $25,00 \%$ & $14,38 \%$ & $13,88 \%$ & $17,71 \%$ & $25,00 \%$ & $19,19 \%$ \\
\hline FC Accesibilidad & $25,00 \%$ & $30,63 \%$ & $17,83 \%$ & $40,63 \%$ & $25,00 \%$ & $27,82 \%$ \\
\hline FC Anegamiento & $25,00 \%$ & $24,38 \%$ & $13,88 \%$ & $17,71 \%$ & $25,00 \%$ & $21,19 \%$ \\
\hline FC Precipitación & $25,00 \%$ & $30,63 \%$ & $54,41 \%$ & $23,96 \%$ & $25,00 \%$ & $31,80 \%$ \\
\hline Razón de consistencia & 0,00 & 0,06 & 0,05 & 0,06 & 0,00 & \\
\hline
\end{tabular}

Elaboración propia, 2020.

Con los valores promedio obtenidos en cada uno de los tres niveles, se procede a promediarlos nuevamente, pero esta vez entre los niveles, con el fin de establecer una media definitiva del valor de cada uno de los factores. 
Tabla 15. Promedio de las ponderaciones en los tres niveles para los factores de la categoría física

\begin{tabular}{|c|c|c|c|c|}
\hline CATEGORÍAS & SUI VALIDATIO & SCIENTATIS VALIDATIO & SOCIETATIS VALIDATIO & PROMEDIO \\
\hline FC Erodabilidad & $25 \%$ & $37,86 \%$ & $19,19 \%$ & $27,35 \%$ \\
\hline FC Accesibilidad & $25 \%$ & $21,85 \%$ & $27,82 \%$ & $24,89 \%$ \\
\hline FC Anegamiento & $25 \%$ & $21,85 \%$ & $21,19 \%$ & $22,68 \%$ \\
\hline FC Precipitación & $25 \%$ & $18,45 \%$ & $31,80 \%$ & $25,08 \%$ \\
\hline
\end{tabular}

Elaboración propia, 2020.

\subsection{Ponderación de los factores de corrección - categoría ecológica}

Se repitió el ejercicio con la participación de las mismas personas que para la ponderación anterior (categoría física), obteniendo los siguientes valores:

Tabla 16. Ponderación nivel Sui Validatio de los factores de corrección de la categoría ecológica

\begin{tabular}{|c|c|}
\hline FACTORES DE CORRECCIÓN & GRUPO INVESTIGACIÓN \\
\hline FC Fauna & $15,78 \%$ \\
\hline FC Flora & $18,67 \%$ \\
\hline FC retamo espinoso & $65,55 \%$ \\
\hline Razón de consistencia & 0.04 \\
\hline
\end{tabular}

Elaboración propia, 2020.

Tabla 17. Ponderación nivel Scientatis Validatio de los factores de corrección de la categoría ecológica

\begin{tabular}{|c|c|c|c|}
\hline FACTORES DE CORRECCIÓN & $\begin{array}{c}\text { EXPERTO } \\
\mathbf{1}\end{array}$ & $\begin{array}{c}\text { EXPERTO } \\
\mathbf{2}\end{array}$ & PROMEDIO \\
\hline FC Fauna & $15,78 \%$ & $18,24 \%$ & $17,01 \%$ \\
\hline FC Flora & $18,67 \%$ & $14,54 \%$ & $16,61 \%$ \\
\hline FC retamo espinoso & $65,55 \%$ & $67,22 \%$ & $66,39 \%$ \\
\hline Razón de consistencia & 0,04 & 0,08 & \\
\hline
\end{tabular}

Elaboración propia, 2020.

Tabla 18. Ponderación nivel Societatis Validatio de los factores de corrección de la categoría ecológica

\begin{tabular}{|c|c|c|c|c|c|c|}
\hline $\begin{array}{c}\text { FACTORES DE } \\
\text { CORRECCIÓN }\end{array}$ & VISITANTE 1 & VISITANTE 2 & VISITANTE 3 & VISITANTE 4 & VISITANTE 5 & PROMEDIO \\
\hline FC Fauna & $33,33 \%$ & $58,89 \%$ & $42,86 \%$ & $60,00 \%$ & $33,33 \%$ & $45,68 \%$ \\
\hline FC Flora & $33,33 \%$ & $25,19 \%$ & $42,86 \%$ & $20,00 \%$ & $33,33 \%$ & $30,94 \%$ \\
\hline $\begin{array}{c}\text { FC retamo } \\
\text { espinoso }\end{array}$ & $33,33 \%$ & $15,93 \%$ & $14,29 \%$ & $20,00 \%$ & $33,33 \%$ & $23,38 \%$ \\
\hline $\begin{array}{c}\text { Razón de } \\
\text { consistencia }\end{array}$ & 0,00 & 0,07 & 0,00 & 0,00 & 0,00 & \\
\hline
\end{tabular}

Elaboración propia, 2020. 
A continuación, se muestran los resultados de las ponderaciones en los tres niveles para los factores de fauna, flora y retamo espinoso, pertenecientes a la categoría ecológica.

Tabla 19. Promedio de las ponderaciones en los tres niveles para los factores de la categoría ecológica

\begin{tabular}{|c|c|c|c|c|}
\hline CATEGORÍAS & SUI VALIDATIO & SCIENTATIS VALIDATIO & SOCIETATIS VALIDATIO & PROMEDIO \\
\hline FC Fauna & $15,78 \%$ & $17,01 \%$ & $45,68 \%$ & $26,16 \%$ \\
\hline FC Flora & $18,67 \%$ & $16,61 \%$ & $30,94 \%$ & $22,07 \%$ \\
\hline FC retamo espinoso & $65,55 \%$ & $66,39 \%$ & $23,38 \%$ & $51,77 \%$ \\
\hline
\end{tabular}

Elaboración propia, 2020.

\subsection{Ponderación Factores categoría social} $100 \%$.

En esta categoría únicamente se incluyó un factor, por lo que el peso del mismo es del

\subsubsection{Cálculo de la Capacidad De Carga Real (CCR) con la metodología CATUM}

Una vez se obtienen los pesos específicos para los factores de corrección, se procede a realizar el cálculo de la capacidad de carga real con la metodología de Capacidad de Acogida Turística Multicriterio para los 5 senderos con los que cuenta la reserva El Encenillo.

De acuerdo al promedio aritmético de los pesos obtenidos para cada uno de los factores mediante la aplicación del proceso jerárquico analitico (AHP) y la metodología 3's, se calcula a continuación el valor del factor de corrección, para lo cual se realiza una multiplicación del peso por el valor del factor; proceso que se realiza para las tres categorías: física, ecológica y social (ver tabla 20, 21 y 22)

Tabla 20. Cálculo del factor de corrección ponderado por categoría física

\begin{tabular}{|c|c|c|c|c|}
\hline SENDERO & CATEGORÍAS & PESO & $\begin{array}{l}\text { VALOR DEL } \\
\text { FACTOR }\end{array}$ & $\begin{array}{l}\text { VALOR } \\
\text { FINAL }\end{array}$ \\
\hline \multirow{5}{*}{ COLIBRI } & FC Erodabilidad & $27,35 \%$ & 0,899 & 0,25 \\
\hline & FC Accesibilidad & $24,89 \%$ & 1,000 & 0,25 \\
\hline & FC Anegamiento & $22,68 \%$ & 0,989 & 0,22 \\
\hline & FC Precipitación & $25,08 \%$ & 0,452 & 0,11 \\
\hline & \multicolumn{3}{|c|}{ Factor de corrección por la categoría física } & 0,83 \\
\hline \multirow{4}{*}{ ORQUIDEAS } & FC Erodabilidad & $27,35 \%$ & 0,973 & 0,27 \\
\hline & FC Accesibilidad & $24,89 \%$ & 0,989 & 0,25 \\
\hline & FC Anegamiento & $22,68 \%$ & 0,997 & 0,22 \\
\hline & FC Precipitación & $25,08 \%$ & 0,452 & 0,11 \\
\hline
\end{tabular}




\begin{tabular}{|c|c|c|c|c|}
\hline \multirow{5}{*}{ MIRADOR } & FC Erodabilidad & $27,00 \%$ & 0,971 & 0,26 \\
\hline & FC Accesibilidad & $24,89 \%$ & 0,963 & 0,24 \\
\hline & FC Anegamiento & $22,68 \%$ & 0,993 & 0,23 \\
\hline & FC Precipitación & $25,08 \%$ & 0,452 & 0,11 \\
\hline & \multicolumn{3}{|c|}{ Factor de corrección por la categoría física } & 0,84 \\
\hline \multirow{5}{*}{ CUSUMBO } & FC Erodabilidad & $27,00 \%$ & 0,986 & 0,27 \\
\hline & FC Accesibilidad & $24,89 \%$ & 1,000 & 0,25 \\
\hline & FC Anegamiento & $22,68 \%$ & 0,991 & 0,22 \\
\hline & FC Precipitación & $25,08 \%$ & 0,452 & 0,11 \\
\hline & \multicolumn{3}{|c|}{ Factor de corrección por la categoría física } & 0,85 \\
\hline \multirow{5}{*}{ VAN DER HAMMEN } & FC Erodabilidad & $27,00 \%$ & 0,986 & 0,27 \\
\hline & FC Accesibilidad & $24,89 \%$ & 1,000 & 0,25 \\
\hline & FC Anegamiento & $22,68 \%$ & 0,991 & 0,22 \\
\hline & FC Precipitación & $25,08 \%$ & 0,452 & 0,11 \\
\hline & \multicolumn{3}{|c|}{ Factor de corrección por la categoría física } & 0,85 \\
\hline
\end{tabular}

Elaboración propia, 2020.

Tabla 21. Cálculo del factor de corrección ponderado por categoría ecológica

\begin{tabular}{|c|c|c|c|c|}
\hline SENDERO & FACTOR DE CORRECCIÓN & PESO & VALOR DEL FACTOR & VALOR FINAL \\
\hline \multirow{4}{*}{ COLIBRI } & FC Fauna & $26,16 \%$ & 0,8383 & 0,22 \\
\hline & FC Flora & $22,07 \%$ & 1,0000 & 0,22 \\
\hline & FC retamo espinoso & $51,77 \%$ & 0,6739 & 0,35 \\
\hline & \multicolumn{3}{|c|}{ Factor de corrección por la categoría ecológica } & 0,79 \\
\hline \multirow{4}{*}{ ORQUIDEAS } & FC Fauna & $26,16 \%$ & 0,7611 & 0,20 \\
\hline & FC Flora & $22,07 \%$ & 0,4556 & 0,10 \\
\hline & FC retamo espinoso & $51,77 \%$ & 0,7260 & 0,38 \\
\hline & \multicolumn{3}{|c|}{ Factor de corrección por la categoría ecológica } & 0,68 \\
\hline \multirow{4}{*}{ MIRADOR } & FC Fauna & $26,16 \%$ & 0,8059 & 0,22 \\
\hline & FC Flora & $22,07 \%$ & 0,8059 & 0,18 \\
\hline & FC retamo espinoso & $51,77 \%$ & 0,1354 & 0,07 \\
\hline & \multicolumn{3}{|c|}{ Factor de corrección por la categoría ecológica } & 0,47 \\
\hline \multirow{4}{*}{ CUSUMBO } & FC Fauna & $26,16 \%$ & 0,7611 & 0,24 \\
\hline & FC Flora & $22,07 \%$ & 0,6930 & 0,15 \\
\hline & FC retamo espinoso & $51,77 \%$ & 0,7127 & 0,37 \\
\hline & \multicolumn{3}{|c|}{ Factor de corrección por la categoría física } & 0,72 \\
\hline \multirow{4}{*}{ VAN DER HAMMEN } & FC Fauna & $26,16 \%$ & 0,8986 & 0,24 \\
\hline & FC Flora & $22,07 \%$ & 0,9237 & 0,20 \\
\hline & FC retamo espinoso & $51,77 \%$ & 0,9237 & 0,48 \\
\hline & \multicolumn{3}{|c|}{ Factor de corrección por la categoría ecológica } & 0,92 \\
\hline
\end{tabular}

Elaboración propia, 2020. 
Tabla 22. Cálculo del factor de corrección ponderado por categoría social

\begin{tabular}{|c|c|c|c|c|}
\hline SENDERO & FACTOR DE CORRECCIÓN & PESO & VALOR DEL FACTOR & VALOR FINAL \\
\hline \multirow{2}{*}{ COLIBRI } & FC Social & $100 \%$ & 0,0364 & 0,04 \\
\hline & \multicolumn{3}{|c|}{ Factor de corrección por la categoría social } & 0,04 \\
\hline \multirow{2}{*}{ ORQUÍDEAS } & FC Social & $100 \%$ & 0,0364 & 0,04 \\
\hline & \multicolumn{3}{|c|}{ Factor de corrección por la categoría social } & 0,04 \\
\hline \multirow{2}{*}{ MIRADOR } & FC Social & $100 \%$ & 0,0364 & 0,04 \\
\hline & \multicolumn{3}{|c|}{ Factor de corrección por la categoría social } & 0,04 \\
\hline \multirow{2}{*}{ CUSUMBO } & FC Social & $100 \%$ & 0,0364 & 0,04 \\
\hline & \multicolumn{3}{|c|}{ Factor de corrección por la categoría social } & 0,04 \\
\hline \multirow{2}{*}{ VAN DER HAMMEN } & FC Social & $100 \%$ & 0,0364 & 0,04 \\
\hline & \multicolumn{3}{|c|}{ Factor de corrección por la categoría social } & 0,04 \\
\hline
\end{tabular}

Elaboración propia, 2020.

Con el valor de los factores de las categorías: Física, ecológica y social, se calcula la capacidad de carga real, para el sendero Colibrí (este proceso se repite con cada uno de los senderos) (ver tabla No. 23).

Tabla 23. Cálculo capacidad de carga real - 5 senderos

\begin{tabular}{|c|c|c|c|c|c|}
\hline SENDERO & CCF & $\begin{array}{c}\text { FC CATEGORIA } \\
\text { FÍSICA }\end{array}$ & $\begin{array}{c}\text { FC CATEGORIA } \\
\text { ECOLÓGICA }\end{array}$ & $\begin{array}{c}\text { FC CATEGORIA } \\
\text { SOCIAL }\end{array}$ & $\begin{array}{c}\text { CAPACIDAD DE } \\
\text { CARGA REAL }\end{array}$ \\
\hline Colibrí & 2747,452 & 0,83 & 0,79 & 0,04 & $\mathbf{7 2}$ \\
\hline Orquídeas & 4713,75 & 0,85 & 0.68 & 0,04 & $\mathbf{1 0 9}$ \\
\hline Mirador & 3490 & 0,84 & 0,47 & 0,04 & $\mathbf{5 5}$ \\
\hline Cusumbo & 4065.72 & 0,85 & 0,72 & 0,04 & $\mathbf{1 0 0}$ \\
\hline $\begin{array}{c}\text { Van der } \\
\text { Hammen }\end{array}$ & 5501.22 & 0,85 & 0,92 & 0,04 & $\mathbf{1 7 2}$ \\
\hline
\end{tabular}

Elaboración propia, 2020.

\subsubsection{Capacidad de Carga Efectiva}

A continuación, se presentan los resultados de la capacidad de carga efectiva, para lo cual debió calcularse la capacidad de manejo de la Reserva el Encenillo (Ver Tabla No. 10) y multiplicarla por la Capacidad de Carga Real para los 5 senderos (Ver Tabla No. 23).

\subsubsection{Capacidad de manejo}

Los valores de la capacidad de manejo son iguales que los obtenidos para la Capacidad de Carga Turística metodología Cifuentes (Ver Tabla No. 10). Con el valor de la capacidad de manejo, se procede a calcular efectivamente cuantos son los turistas que pueden ingresar a la Reserva El Encenillo de acuerdo con la metodología de CATUM (Ver Tabla No. 24). 
Tabla 24, Cálculo capacidad de carga efectiva metodología CATUM

\begin{tabular}{|c|c|c|c|}
\hline SENDERO & $\begin{array}{c}\text { PORCENTAJE DE } \\
\text { CAPACIDAD DE MANEJO }\end{array}$ & $\begin{array}{c}\text { CAPACIDAD DE CARGA } \\
\text { REAL }\end{array}$ & $\begin{array}{c}\text { CAPACIDAD DE CARGA } \\
\text { EFECTIVA- } \\
\text { CCT }\end{array}$ \\
\hline Colibrí & $63 \%$ & 72 & 45 \\
\hline Orquídeas & $63 \%$ & 109 & 69 \\
\hline Mirador & $63 \%$ & 55 & 35 \\
\hline Cusumbo & $63 \%$ & 100 & 63 \\
\hline Van der Hammen & $63 \%$ & 172 & 108 \\
\hline
\end{tabular}

Elaboración propia, 2020.

\subsection{Comparación de resultados metodologías Capacidad de Carga Turística y Capacidad de Acogida Turística Multicriterio}

A continuación, se presentan los valores de la capacidad de carga efectiva con ambas metodologías (Capacidad de Carga Turística - CCT y Capacidad de Acogida Turística Multicriterio - CATUM).

Tabla 25. Cálculo capacidad de carga efectiva metodología CCT y CATUM

\begin{tabular}{|c|c|c|c|}
\hline SENDERO & $\begin{array}{c}\text { CAPACIDAD DE CARGA } \\
\text { EFECTIVA } \\
\text { CCT }\end{array}$ & $\begin{array}{c}\text { CAPACIDAD DE CARGA } \\
\text { EFECTIVA } \\
\text { CATUM }\end{array}$ & $\begin{array}{c}\text { NUMERO DE } \\
\text { VISITANTES DE } \\
\text { DIFERENCIA }\end{array}$ \\
\hline Colibrí & 7 & 45 & $\mathbf{3 8}$ \\
\hline Orquídeas & 4 & 69 & $\mathbf{6 5}$ \\
\hline Mirador & 20 & 35 & $\mathbf{1 5}$ \\
\hline Cusumbo & 6 & 63 & $\mathbf{5 7}$ \\
\hline Van der Hammen & 17 & 108 & $\mathbf{9 1}$ \\
\hline
\end{tabular}

Elaboración propia, 2020.

De acuerdo a los resultados de la tabla 25, se observa que la Capacidad de Carga Turística calculada de acuerdo a la metodología descrita por Cifuentes es bastante restrictiva en cuanto a visitantes y arroja números permitidos por día, para 3 de los 5 senderos inferiores a las personas que se manejan en la actualidad por cada grupo (el cual es de 17 personas), siendo este valor poco viable económicamente, y no sostenible en el tiempo. Se observa en el valor que arroja el cálculo con la metodología CATUM, que presenta números más cercanos a la realidad del manejo que se le está dando al turismo en la reserva, sin embargo, como se menciono en el apartado 5.1.3.1. capacidad de manejo, es necesario que la reserva mejoré la infraestructura para la prestación del servicio turístico, al igual que el equipamiento con el que se cuenta, y el personal, en cuanto a número contratado y competencias necesarias para que guie al visitante; lo cual, permitirá mejorar la calidad de la experiencia del visitante, y poder manejar valores de visitantes más altos de acuerdo a la CATUM.

Es importante señalar que en este momento no se evidencien impactos negativos significativos derivados de la actividad turística y que puedan afectar significativamente los 
objetivos de conservación del área, sin embargo, se deben establecer indicadores para realizar el monitoreo y seguimiento, de forma que se pueda establecer con criterios adicionales cual es el verdadero valor de la capacidad de carga de la reserva, el arrojado por la CCT o el que proporciona la CATUM.

Tener en cuenta 8 factores de corrección con la metodología de Capacidad de Carga Turística usada por Cifuentes es un número demasiado elevado y elimina casi en su totalidad la posibilidad de realizar turismo en este tipo de áreas, sin considerarse que no se tienen en cuenta todas las variables que debería incluir este tipo de estudio. Con la propuesta de Capacidad de Acogida Turística Multicriterio - CATUM, se pueden tener en cuenta 8 variables o más; siendo aconsejable que se adicionen otros factores de corrección, en las tres categorías de la sostenibilidad: ecológica, económica y social.

\section{CONCLUSIONES}

La metodología CATUM, incluye desde su conceptualización elementos de la planeación y gestión de la actividad turistica sustanciales como lo es el pensar en el territorio receptor de turismo, sin embargo, en la presente investigación, por ser una comparación de resultados utilizando los mismos factores de corrección, no se logra mostrar con claridad esta inclusión que se realiza.

De acuerdo a lo anterior, se sugiere incluir para futuros estudios una descripción del territorio y factores de corrección adicionales en la categoría ambiental, en específico para la reserva El Encenillo, se recomienda el factor de nidificación de las aves (cuando son más sensibles a las perturbaciones), en la económica se proponen: la percepción de gasto del turista y percepción de ingresos de la reserva, y factores adicionales en la social como: satisfacción del turista y satisfacción de la reserva y de la comunidad aledaña a la misma, de forma que sea aún más robusto el cálculo de los visitantes que se pueden recibir. De igual forma, se recomienda que se ponderen las categorías a las que pertenecen los factores, de manera que se realice un análisis en niveles, elemento que permite consolidar el cálculo y la metodología de AHP lo permite.

Realizar la ponderación de los pesos con 3 niveles de participación (Sui, Scienciatis y Societatis Validatio) dota al proceso de cálculo de los pesos de los factores, que son utilizados para calcular el número máximo de visitantes, de menor subjetividad y permite obtener un consenso entre los investigadores, administradores de las áreas protegidas, los turistas y en lugares donde se presente de la propia comunidad local; no obstante, se aconseja que se utilice un mayor número de expertos y de visitantes, de forma que se haga representativa la muestra, lo cual significa que se debe tener mayor disponibilidad de tiempo y presupuesto para este tipo de estudios.

De acuerdo a lo anterior, se puede concluir que un estudio con la metodología CATUM es más demorado, requiere de mayor conocimiento y una mayor participación de administradores, expertos y visitantes, que el cálculo realizado con la metodología de Capacidad de Carga Turística, pero a su vez permite disminuir la subjetividad y fortalecer la robustez de los resultados. 
Por último, el número máximo de turistas en un área natural, no es un número mágico que conlleve inmediatamente a la realización de turismo sostenible, éste debe ir acompañado de herramientas como lo son los planes de manejo, en donde un mayor flujo de visitantes pueda ser gestionado adecuadamente. De igual forma se aconseja realizar monitoreo y seguimiento que los impactos que la actividad turística origina sobre las zonas utilizadas para éste fin, de forma que se determine si efectivamente la capacidad de carga calculada, permite cumplir con los objetivos de conservación de estos espacios.

\section{REFERENCIAS}

Aguilar-Garavito, M. (2010). Restauración ecológica de áreas afectadas por Ulex europaeus, Serranía El Zuque, Reserva Forestal Protectora Bosque Oriental de Bogotá, localidad 4 San Cristóbal, Bogotá D.C., Colombia. Bogotá D.C.: Convenio 005/09 SDA-IDIPRON. 82 pp.

Alipour, H, Altinay, M., Hussain, K. y Sheikhani, N. (2007). Perceptions of the beach users: a case study of the coastal areas of North Cyprus towards establishment of a 'carrying capacity', FIU Review, 24 (2), 28-48.

Álvarez, M. P. (2010). Evaluación de la capacidad de carga: Una herramienta para el manejo y la conservación de los sitios patrimoniales. Canto Rodado: Revista especializada en patrimonio, (5), 213-240.

Amador, E., Cayot, L., Cifuentes, M., Cruz, E., Cruz, F., \& Ayora, P. (1996). Determinación de la capacidad de carga turística en los sitios de visita del Parque Nacional Galápagos. Servicio Parque Nacional Galápagos, Ecuador. 42p.

Ardila, J. D. G., Suarez, A. S., \& Fernández, F. G. (2016). Cálculo de los Límites de Cambio Aceptable (LAC) en el sendero lagunas de Siecha, parque nacional natural ChingazaColombia. Revista de Tecnología, 15(2), 75-88.

Baptiste, M., N. Castaño, D. Cárdenas, F. Gutiérrez, D. Gil, D. y C. A. Lasso. (2010). Análisis de riesgo y propuesta de categorización de especies introducidas para Colombia. Bogotá D.C.: Instituto de Investigación de Recursos Biológicos Alexander von Humboldt. 200 pp.

Barrera, J., Ríos, H., \& Pinzón, C. (2002). Planteamiento de la propuesta de restauración ecológica de áreas afectadas por el fuego y/o invadidas por el retamo espinoso. Ulex europaeus, 55-71.

Barrera Tolosa, L. H. (2012). Diseño de los lineamientos de política del servicio de policía ambiental para la Policía Nacional de Colombia (MS thesis). Universidad de La Sabana, Chía - Colombia

Beltrán-G, H. \& Barrera-Cataño, J. I. (2014). Caracterización de invasiones de Ulex europaeus L. de diferentes edades como herramienta para la restauración ecológica de bosques altoandinos, Colombia. Biota Colombiana, v. 15.

Bentz, J., Lopes, F., Calado, H., \& Dearden, P. (2016). Sustaining marine wildlife tourism through linking Limits of Acceptable Change and zoning in the Wildlife Tourism Model. Marine Policy, 68, 100-107. 
Bertoni, M; López, M. J. y Testa, J. (2016). La escala sustentable de los destinos turísticos. Una revisión crítica de la capacidad de carga. Comunicación presentada en 1 Jornadas de Hábitat y Ambiente, Mar del Plata - Argentina.

Berumen, S. A., \& Redondo, F. L. (2007). La utilidad de los métodos de decisión multicriterio (como el AHP) en un entorno de competitividad creciente. Cuadernos de administración, 20(34), 65-87.

Botero Saltarén, C., Hurtado García, Y., González Porto, J., Ojeda Manjarrés, M., \& Díaz Rocca, L. H. (2008). Metodología de cálculo de la capacidad de carga turística como herramienta para la gestión ambiental y su aplicación en cinco playas del caribe norte Colombiano. Gestión y Ambiente, 11(3).

Buckley, R. (1999). An ecological perspective on carrying capacity, Annals of Tourism Research, 26 (3), 705-708

Butler, R.V. (1997). The concept of carrying capacity for tourism destinations: dead or merely buried? En: C. Cooper y S. Wanhill (Eds.), Tourism development: environmental and community issues (pp. 11-21), Chichester: John Wiley \& Sons.

Canadian Arctic Resources Committee (2002), Carrying capacity and thresholds: theory and practice in environmental management, Macleod Institute, Calgary.

Cifuentes, M., Alpizar, W., Barroso, F., Courrau, J., Falck, M. L., Jiménez, R \& Tejada, J. (1990). Capacidad de Carga Turística de la Reserva Biológica Carara. Turrialba, Costa Rica.

Cifuentes, M. (1992a). Determinación de capacidad de carga turística en áreas protegidas (No. 194). Bib. Orton IICA/CATIE.

Cifuentes Arias, M. (1992b). Determinación de capacidad de carga turística en áreas protegidas (No. 333.95 C569d). Turrialba, CR: CATIE.

Cifuentes Arias, M., Mesquita, C. A. B., Méndez, J., Morales, M. E., \& Aguilar, N. (1999). Capacidad de carga turística de las áreas de uso público del Monumento Nacional Guayabo, Costa Rica (No. 338.4791 C236c). Turrialba, CR: WWF.

Clements, D. R., D. J. Peterson y R. Prasad. (2001). The biology of Canadian weeds. 112. Ulex europaeus. Canadian Journal of Plant Science 81: 325-337.

Cloquell, V. (2003). Propuesta metodológica para la validación previa de indicadores y funciones de valor en el problema unificado de localización y evaluación del impacto ambiental de proyectos (Tesis doctoral). Universidad Politécnica de Valencia.

Cloquell-Ballester, V. A., Cloquell-Ballester, V. A., Monterde-Diaz, R., \& Santamarina-Siurana, M. C. (2006). Indicators validation for the improvement of environmental and social impact quantitative assessment. Environmental Impact Assessment Review, 26(1), 79-105

Galacho-Jiménez, F. B., \& Castaño, J. A. (2008). El modelo de evaluación de la capacidad de acogida del territorio. Aspectos conceptuales y técnicas relacionadas. Investigaciones Geográficas, 60. julio-diciembre de 2013, pp. 69 - 85. DOI: 10.14198/INGEO2013.60.04

García-Hernández, M. (2000). Turismo y medio ambiente en ciudades históricas. De la capacidad de acogida turística a la gestión de los flujos de visitantes. Anales de Geografía de la Universidad Complutense, 20. Pp. 131-148. ISSN: 0211-9803.

Gómez, J, D., Sánchez, A \& Gutiérrez-Fernández, F. (2016). Cálculo de los límites de cambio aceptable (LAC) en el sendero Lagunas de Siecha, Parque Nacional Natural Chingaza Colombia. Revista de Tecnología, 15(2), 75-88. 
Gómez Orea, D. 1992. Ordenación Rural. Ed. Agrícola Española-Ministerio de Agricultura Pesca y Alimentación. $396 \mathrm{p}$

Graefe, A.R., Kuss, F.R. and Vaske, J.J. (1990) Visitor Impact Management: A Review of Research. National Parks and Conservation Association, Washington DC

Gutiérrez-Fernández, L. F. (2011). Diseño y validación de un sistema de indicadores de sostenibilidad para la evaluación de áreas naturales con uso turístico y su aplicación al territorio colombiano (Doctoral dissertation, Universitat Politècnica de Valencia).

Gutiérrez-Fernández, F., Cloquell Ballester, V., \& Cloquell Ballester, V. (2012). Propuesta de un sistema de indicadores de sostenibilidad para áreas naturales con uso turístico, validado mediante consulta a terceros. Turismo Y Sociedad, 13, 55-83. Recuperado a partir de https://revistas.uexternado.edu.co/index.php/tursoc/article/view/3391

Gutiérrez, F. G., \& Sierra, S. A. (2015). Cálculo de la capacidad de carga turística del lago Tarapoto-Puerto Nariño (Amazonas-Colombia). Revista de Tecnología, 14(1), 85-96.

Gutiérrez-Fernández, Fernando, \& Rodríguez-Torres, Sandra. (2019). Cálculo de acogida turística multicriterio (catum), caso de estudio Haynes cay, ubicado en el Archipelago de San Andrés- Colombia (reserva mundial de la biósfera - "seaflower"). Revista interamericana de ambiente y turismo, 15(1), 28-45. https://dx.doi.org/10.4067/ $\underline{\mathrm{S} 0718-235 \times 2019000100028}$

Ibañez-Pérez, R. (2016). Capacidad de carga turística como base para el manejo sustentable de actividades ecoturísticas en Unidades de Manejo Ambiental (UMA) de Baja California Sur (BCS)". El periplo sustentable, (30), 37-76

Kaal, J., A. Martínez-Cortizas, O. Reyes y M. Soliño. (2012). Molecular characterization of Ulex europaeus biochar obtained from laboratory heat treatment experiments - A pyrolysis-GC/MS study. Journal of Analytical and Applied Pyrolysis 95: 205-212.

Lindberg, K., Mccool, S. y Stankey, G. (1997). Rethinking carrying capacity, Annals of Tourism Research, 24 (2), 461-465.

López-Bonilla, L. M., \& López-Bonilla, J. M. (2008). La capacidad de carga turística: revisión crítica de un instrumento de medida de sostenibilidad. El Periplo Sustentable, 15, 123-150

Lowe, S., M. Browne, S. Boudjelas y M. De Poorter. (2000). 100 of the World's Worst Invasive Alien Species. A selection from the Global Invasive Species Database. Auckland, New Zealand: The Invasive Species Specialist Group ISSG a specialist group of the Species Survival Commission SSC of the World Conservation Union IUCN.

Maggi, E; Fredella, F. L. (2010). The carrying capacity of a tourist destination. The case of a coastal Italian city, 50th Congress of the European Regional Science Association: "Sustainable Regional Growth and Development in the Creative Knowledge Economy", 19-23 August 2010, Jönköping, Sweden, European Regional Science Association (ERSA), Louvain-la-Neuve.

Marín-Santamaría, C. M. (2010). Potencial de aprovechamiento alimenticio para consumo humano de frutos silvestres en la Reserva Biológica Encenillo, Guasca, Cundinamarca, Pontificia Universidad Javeriana (Trabajo de pregrado).

Martínez X, Rincón D, Galvis P, Monje C. (2005). Valoración biofisíca y planificación predial para la conformación de la Reserva Encenillo, Guasca - Cundinamarca. Informe Fundación Natura. P. 73. 
McCool, S. y Lime, D. (2001). Tourism carrying capacity: tempting fantasy or useful reality? Journal of Sustainable Tourism, 9 (5), 372-388.

McCool, S.F. 2013. Limits of Acceptable Change and Tourism In Holden, A., and Fennel, D.A. (eds) Routledge Handbook of Tourism and the Environment. Routledge. Oxon, UK. Pp 285-298.

Medel, R. G., \& Jaksic, F. M. (1988). Ecología de los cánidos sudamericanos: una revisión. Revista chilena de historia natural, 61(1), 67-79.

Montalvo, R. \& Castillo, J. J. (2018). Estimación de la capacidad de carga turística en Agua Selva (Tabasco - México), base para la planificación y el desarrollo regional. Estudios y Perspectivas en Turismo, vol. 27, núm. 2. Pp. 295-31

Moore, J. (2006). Controlling gorse seed banks. 15th Australian Weeds Conference proceedings: managing weeds in a changing climate. Australia: The Weed Management Society of South Australia. Pp. 283-286.

Morales-Betancourt, D. (2014). Capacidad De Carga Física Y Real Para Atractivos Turístico Priorizados Y Vías De Acceso En El Casco Urbano De Puerto Nariño, Amazonas. Anuario Turismo y Sociedad, 15.

Moreno-Jiménez, M. (2002). El proceso analítico jerárquico (AHP). Fundamentos, metodología y aplicaciones. Revista Electrónica de Comunicaciones y Trabajos de ASEPUMA, 1, 28-77.

Navarro, M; Tejada, F; Almeida, J; Cabello, R; Cortés, J; Delgado, F; Fernández, G; Gutiérrez, M. Luque, G. Málvarez, O; Marcenaro, F; Navas, F; Ruiz de la Rúa, J; Ruiz, F; Solís. (2012). Carrying capacity assessment for tourist destinations. Methodology for the creation of synthetic indicators applied in a coastal area, Tourism Management, Volume 33, Issue 6. Pages 1337-1346. https://doi.org/10.1016/j.tourman.2011.12.017.

Ocampo-Zuleta, K., \& Solorza-Bejarano, J. (2017). Banco de semillas de retamo espinoso Ulex europaeus $\mathrm{L}$. en bordes del matorral invasor en un ecosistema zonal de bosque altoandino, Colombia. Biota Colombiana, 18(1 Sup), 89-98.

OMT. (1981). Saturation of tourist destinations: Report of the secretary general. Madrid -España.

OMT. (2005). Indicadores de desarrollo sostenible para los destinos turísticos. Guía práctica. Organización Mundial del Turismo. Madrid. ISBN. 92-844-0838-5

O'Reilly, A. M. (1986). Tourism carrying capacity: concept and issues. Tourism management, 7(4), 254-258.

Ortega, J. L. C., Dagostino, R. M. C., \& Olivas, M. L. B. (2020). Capacidad de carga turística de la Playa Punta Perula y Playa Isla Cocinas como estrategia para un uso turístico sustentable. CIMEXUS, 14(2), 11-26.

PAP/RAC 1997 Guidelines for Carrying Capacity Assessment for Tourism in Mediterranean Coastal Areas. PAP-9/1997/G.1. Split, Priority Actions Programme Regional Activity Centre, viii+51.

Porter, M. (1991). La Ventaja Competitiva de las Naciones. Buenos Aires: Editorial Vergara.

Porter, M.E. (2010). Ventaja competitiva: Creación y sostenibilidad de un rendimiento superior. Madrid: Pirámide.

Rees, M. y R. L. Hill. 2001. Large-scale disturbances, biological control and the dynamics of gorse populations. Journal of Applied Ecology 38: 364-377. 
Rees, W \& Wackernagel, M. (1996). Urban ecological footprints: Why cities cannot be sustainable-And why they are a key to sustainability, Environmental Impact Assessment Review, Volume 16, Issues 4-6. Pages 223-248. https://doi.org/10.1016/ S0195-9255(96)00022-4.

Regil-García, Héctor Hugo, \& Franco-Maass, Sergio. (2009). Nivel de adecuación del territorio para el desarrollo de especies agrícolas y forestales en el Parque Nacional Nevado de Toluca. Economía, sociedad y territorio, 9(31), 803-830.

República de Colombia, Ministerio de Comercio, Industria y Turismo \& Ministerio de Ambiente, Vivienda y Desarrollo Territorial. (2003). Política para el desarrollo del ecoturismo. Bogotá - Colombia

Rodríguez Torres, S. (2018). Propuesta metodológica para la medición de la capacidad de carga turística del Haynes Cay en San Andrés Isla. Universidad Externando. Bogotá -Colombia

Saaty, T. (1980). The analytic hierarchy process (AHP) for decision making. In Kobe, Japan.

Sati, V. P. (2018). Carrying capacity analysis and destination development: a case study of Gangotri tourists/pilgrims' circuit in the Himalaya. Asia Pacific Journal of Tourism Research, 23(3), 312-322.

Segrado Pavón, R. G., Arroyo Arcos, L., Amador Soriano, K., Palma Polanco, M., \& Serrano Barquín, R. D. C. (2015). Hacia un Modelo de Aprovechamiento Turístico Sustentable en Áreas Naturales Protegidas: Estudio de Caso del Parque Natural Chankanaab de Cozumel, México. Pasos: Revista de Turismo y Patrimonio Cultural, 13(1).

Tapia, G. (2014). Turismo sostenible. Introducción y marco financiero. Revista de investigación en modelos financieros, 1.

Trumbic, I. (2017). Tourism carrying capacity in Mediterranean coastal tourist destinations. In The Challenge of Tourism Carrying Capacity Assessment. Pp. 197-216.

Udo, N., M. Tarayre y A. Atlan. (2016). Evolution of germination strategy in the invasive species Ulex europaeus. Plant Ecology 10 (2): 375-385.

Ulloa, D; Rodríguez, A. M. \& Gutiérrez-Fernández, F. (2017). Calculo de los Límites de Cambio Aceptable (LAC) en el casco urbano del municipio de Puerto Nariño-Amazonas, Colombia. Revista de Tecnología, 16(2), 78-89.

Vera-Rebollo, J. F. y Baños-Castiñeira, C. J. (2004). Turismo, territorio y medio ambiente, Papeles de Economía Española, 102, 271-286.

Wall, G. (2020). From carrying capacity to overtourism: a perspective article" Tourism Review, Vol. 75 No. 1, pp. 212-215. https://doi.org/10.1108/TR-08-2019-0356

Wallace, R. B., Alfaro, F., Sainz, L., Ríos-Uzeda, B., \& Noss, A. (s.f.). CAPÍTULO 14, Canidae. Mamíferos medianos y grandes de Bolivia. pp. 379, 386. Recuperado de https:// www.researchgate.net/profile/Robert Wallace7/publication/268578283 Canidae/ links/5482698b0cf2f5dd63a89a79/Canidae.pdf

Watson, G.L. y Kopachevsky, J.P. (1996). Tourist carrying capacity: a critical look the discursive dimension, Progress in Tourism and Hospitality Research, 2 (2), 169-17.

Yupanqui, C. M., 2005. Perfil Bioquímico Sanguíneo Hepático en Coatíes (Nasua Nasua) criados en Cautiverio en el departamento de Lima. Pp. 11-13. 